\title{
Analysing Emergent Dynamics of Evolving Computation in 2D Cellular Automata
}

\author{
John S. McCaskill ${ }^{1(凶)}$ (D) and Norman H. Packard ${ }^{1,2}$ (D) \\ ${ }^{1}$ European Centre for Living Technology (ECLT), Ca' Bottacin, Dorsoduro \\ 3911, Calle Crosera, 30123 Venice, Italy \\ johnsmccaskill@gmail.com \\ 2 Daptics Inc., San Francisco, CA, USA
}

\begin{abstract}
Conway's Game of Life (GoL), a famous 2D cellular automaton (CA), is extended to allow evolution by associating genetic information with individual live cells, that specifies variant local CA rules. Genomes are formed by copying (potentially with mutation) or movement from one of the live neighbour cells and are destroyed at death. Just as biological evolution discovers innovations in the space of chemical and physical functionalities, we explore how the addition of genetic information enables an evolutionary process that can coordinate robust complex dynamics by exploring spatially inhomogeneous local modifications to the non-robust GoL rules.

We discovered a large family of deterministic rules which avoid stochastic choices of ancestor for genetic inheritance. Systematic genetic variations near to the game of life rule are investigated and found to produce signs of computational complexity with an abundance of spaceship and glider gun structures. We investigated evolution for four successively more differentiated symmetry cases in the nearest neighbour rules: semi-totalistic, corner-edge totalistic, 8-rotation symmetric, and physical 2D symmetric (4-rotations and 4-reflections).

The genetic evolution is analysed by fast ongoing genealogy construction and population weighted activity statistics. The spatial structure is captured using hash encoded quadtrees of the connected components, which are also mapped through time for novelty and with activity statistics. This together with a novel genetic tracking of the dynamical displacement ancestry of live genes allows an efficient recognition of regular dynamical structures such as spaceships which transport information while changing shape, solving an open problem in finding efficient alternatives to $\varepsilon$-machines for $2 \mathrm{D}$ automata.
\end{abstract}

Keywords: Evolution $\cdot$ Cellular automata $\cdot$ Artificial life $\cdot$ Genealogies $\cdot$ Selforganization - Game of Life $\cdot$ Activity statistics

\section{Introduction}

Interest in cellular automata (CAs), as models of emergent complexity, began with von Neumann's 29-state CA, designed for universal construction [1], and became widespread with Conway's discovery of a simple 2D Game of Life (GoL) [2] and

Electronic supplementary material The online version of this chapter (https://doi.org/10.1007/ 978-3-030-34500-6_1) contains supplementary material, which is available to authorized users.

(C) The Author(s) 2019

C. Martín-Vide et al. (Eds.): TPNC 2019, LNCS 11934, pp. 3-40, 2019.

https://doi.org/10.1007/978-3-030-34500-6_1 
Wolfram's analysis of computational complexity classes in 1D CAs [3], also extended to 2D [4]. Conway's and Von Neumann's life-like CAs are defined with strong relaxation to the ground state in order to facilitate computation by rational design, and universal computation has been proven by construction in both cases [5, 6]. Both however involve fundamentally unprotected computations, in which perturbations, in the form of even the simplest travelling patterns, will almost certainly destroy not only the computed result but the carefully crafted computing architecture as well. Because of this, and despite the widespread continuing interest in novel computational structures in the GoL and related CAs, there is a major jump to evolving systems in which computation needs to survive robustly in the presence of potential interactions with many competitors. This paper is concerned with bridging this gap, maintaining deterministic computation as far as possible, apart from random and rare mutational changes, while supporting locally determined genetically encoded rule changes that enable evolution.

The GoL [2, 7] is a deterministic dynamical system that takes 2D spatial patterns of binary states (1 'live' or 0 'not-alive') on a square lattice to new patterns as time progresses discretely, through the action of a local rule (CA), each site's state at time $t+1$ being dependent on its state and the states of its eight nearest neighbours at time $t$. The game of life is a semi-totalistic cellular automaton (CA) rule, the influence of neighbours on the next state being determined by the sum $s$ of their state values only. A live cell survives only if $s=2$ or 3 , otherwise dying (changing to state 0 ), and a nonlive cell undergoes birth (transitions to 1 ) only if there are exactly 3 live neighbours at time $t$. The GoL has become a canonical example of a complex system, with simple local rules that produce complex dynamics. It has a rich phenomenology of dynamics from special initial conditions, documented in massive catalogue projects and other articles [8]. Specially engineered initial states can have extremely long transients, occupying large regions of space, and indeed the Gol has been shown to support universal computation [5, 9, 10] via a set of motifs including so-called spaceships, glider guns and still-lifes.

Notwithstanding these properties, the GoL has not been a good model for studying the emergence of complexity, for two main reasons. The first is that starting from random initial state patterns on a finite compact domain, it is well known that the GoL almost always settles down to a combination of isolated static and simply periodic structures which are individually of limited spatial extent. Although specially engineered initial states can have extremely long transients, occupying large regions of space, the absence of complex interconnected pattern persistence starting from random initial conditions means that it is not a good candidate for the emergence of complexity. In fact, this behaviour is so robust that, as in a sand-pile, random isolated birth events cause the relaxed state to self-organize to a critical state where there is a power-law of frequencies for cascade magnitudes $[11,12]$. Secondly, the complexity generated by GoL is not robust, in the sense that perturbations destroy functionally complex structures. Even when a complex dynamical structure happens to be produced by a random initial condition, it is typically destroyed by any glider or spaceship that perturbs it.

In biology, genetics is coupled to real-world physics and chemistry, enabling evolution to produce a complex biosphere. In the present work, we use nearest neighbour CAs to provide homogeneous models of rich but simple physical chemistry. 
We then enhance these CAs by including genetic information, with the aim of understanding how complex information may emerge from this simple spatial version of evolution. The coupling of GoL to genetic information has already been attempted in various ways (precursors: immigration, quadlife; with genetics: Sprout Life [13], HetCA [14, 15], evolife [16-19]), but a systematic investigation of evolving dynamics is still outstanding. We add genetics to GoL-like binary CAs by associating a genome with each live cell. The genome of a live cell encodes local departures from the GoL rule, making the system a spatially inhomogeneous cellular automaton. Genetic inheritance is ensured by a newly born live cell's genome being moved from one or copied (potentially with mutation and recombination) from one or more of the live neighbour cells (there are three in the GoL) and being deleted when the cell dies. In this article we focus on the simplest case of mutation and asexual reproduction without recombination. We specify (i) how departures from the GoL are determined by genes; and (ii) how genes are propagated from one time-step to the next, deriving deterministic inheritance rules that may be genetically neutral or sequence dependent. The addition of genetics to the GoL alters long term dynamics; the long-term dynamics of the GoL is a field of static patterns or patterns with low periodicity (blinkers). Figure 1 illustrates such a state, compared with an example of an evolving genelife population with interacting spaceships.
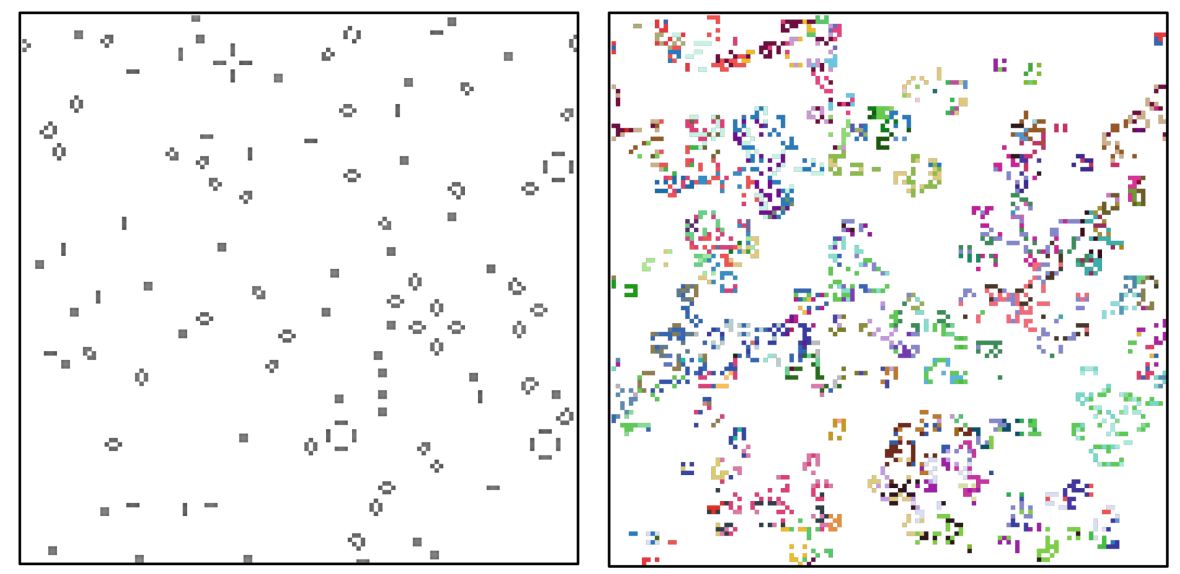

Fig. 1. A comparison of the classic Game of Life's long-time dynamical state after 2000 timesteps (on the left; fixed patterns and simple local periodicity) with an example of an evolving genelife population after the same time (on the right; ongoing generation of novel patterns). For the genelife population, different genomes are shown with different shades of grey (different colours online).

This investigation is motivated by a fundamentally interest in the interaction between computational complexity and evolution. Computational complexity in CAs has addressed the relationship with universal computation, universal construction $[3,4$, $20]$ and the edge of chaos [11, 12], also for the evolution of CAs [21]. Information 
transfer measures of complexity have been applied [22-24] and, most notably, statistical complex dynamics captured with the $\varepsilon$-machine formalism of Crutchfield [25-29]. Complex dynamics have also been addressed in combinatorial game theory [7], with complex spatial pattern formation emerging also in evolving systems [30, 31] including evolutionary games [32, 33].

\section{The Genelife Model}

A genome is associated with each live cell and contains inherited information, copied from its ancestors at birth, which may be used to track the flow of information in both the GoL and the wider family of CA models investigated here. The genome of a live cell encodes the local CA rule governing that cell, and this rule may deviate from the GoL local rule, making the system a spatially inhomogeneous cellular automaton. The local state of a cell is described completely by the presence or absence of a genome (the live/empty (1/0) state in the GoL) and for the case of a live cell, the 64-bit sequence comprising its genome. The restriction to 64-bit binary sequences is not fundamental but enables fast computation using machine integers. Note that it makes more sense to refer to 0 cells as empty (or inanimate) than dead: they contain no bioinformation. Our model could easily be extended to include information strings for the 0 cells (complex resources) as well as the 1 cells; we choose to adhere to the commonly used biomorphic analogy associating life only with the 1 cells.

As in the GoL, we restrict attention to a local CA on a 2D square lattice, with the Moore 8-cell nearest neighbourhood, so that the discrete dynamics are completely defined locally by specifying the next state $c_{i, j}(t+1)$ of a cell at lattice site $(i, j)$ at time $t+1$, in dependence on the previous state of the cell $c_{i, j}(t)$ and of its 8 nearest neighbours. The GoL rule is only semi-totalistic, because its next state depends on the central state in addition to the sum $s_{9}$ of its 9 neighbour states (including the central state), the next state being 1 for $s_{9}==3, c_{i, j}(t)$ for $s_{9}==4$, and 0 otherwise. We employ the 8-neighbour exterior sum $s=s_{8}$ and note that, for the semi-totalistic rules like GoL, the next state is a function of $(c, s)$ where $c=c_{i, j}(t)$. As in natural systems, we retain the distinct dependence on the central state $c$ as well as $s$, but we shall also consider less symmetric rule families which depend on other neighbour properties in addition to their sum $s$. We use throughout the simplifying convention of only specifying rules that result in a 1 (live cell) at the next state, the default being 0 (empty cell).

To complete the specification of the Genetic GoL system dynamics, we need to specify (i) how live/empty next states are determined by the configuration of live neighbours and their genomes (departure from GoL rules are possible), and (ii) how the information in the genomes attached to live states is propagated when the next state is live.

Local Genetically Determined Rules. We use the term genome to refer to the full genetic sequence, reserving the term gene to refer to a specifier of part of the rule-table. How such LUTs are encoded in the genome must be decided by the model. Rather than always to allow all possible rules in a given symmetry model, which may result in ubiquitous proliferation, it is also of interest to consider restricted models in which a 
global constraint is introduced so that only a subset of the possible local states can be specified for active rules, i.e. for gene-dependent modification of the default GoL local rule. For example, in the semi-totalistic case, a birth-survival mask is introduced which would have 1-bits signifying permission for a subset of positions corresponding to particular $s$ values for either survival or birth. While the genes may contain other entries, the model would not allow these genes to enable birth (survival) for local state configurations that correspond to $s$ values for which there is a 0-bit in the birth (survival) mask. For example, with the 16-bit birth-survival mask 0x0406, in the semitotalistic case 2, only at most GoL rules are allowed, and with the mask 0x0606 the genetically encoded extension of the $\mathrm{s}=2,3$ case 1 model family is specified. For the lower symmetry models, the birth-survival mask will contain up to 64 bits. Thus, the mask bits corresponding to birth and survival bits of the genome determine whether the local rule can be affected, according to the local configuration. The globally specified birth and survival masks determine the universe of local rules that will be explored by evolution. Zeros in these masks reduce the size of the universe, ones in the masks enlarge the universe. Exactly how many bits are in the birth and survival masks depend on the degree of symmetry breaking employed in rule construction, as described below. More complex symmetry breaking results in more bits in the mask, generally giving finer grain control of the evolutionary process.

In this work, we consider two types of genetic encodings of rules:

1. a direct position-dependent encoding assigning specific genome bits (or possibly contiguous sets of bits for redundant encodings) at certain positions to specific LUT entries.

2. a modular variable-length position-independent encoding in which the genome encodes (at any block-aligned position) the local states which result in a next state of " 1 ".

With $s$ live neighbours and a centre cell state c ( 0 or 1$)$, there are $s+c$ genomes in the neighbourhood, $s+1$ for survival (and birth with overwrite) transitions and $s$ for pure birth transitions $(0 \rightarrow 1)$. We chose to treat pure birth and overwrite transitions in the same way, and hence in the standard models neglected the central cell's genome influence on overwrite processes, and to keep things uniform also for survival. For survival we did compare this with the natural alternative of only the central genome determining rule departures ( 1 of the option bits in the replication scheme control word). It remained to decide how the $s$ genomes in the neighbourhood should determine the rule departures. Seeking compelling constructions which apply to all possible non-zero $s$ values, we identified three ways of combining the information in the individual genomes for particular LUT entries that should result in a central live cell state at the next time step: (i) And (ii) Or (iii) Majority. "And" means that all, "Or" at least one, of the genomes must contain the LUT transition for it to be effective locally. "Majority" means either $\geq 1 / 2$ and $>1 / 2$ of the genomes must encode a LUT transition for it to be effective. While we have encoded each of these variants in genelife, we concentrate on the case (i) in this paper. We return to the different symmetries of CA rule generalizations, beyond the semi-totalistic rules of the GoL, after explaining the choice of ancestor. 
Choice of Ancestor. Here we address the choice of ancestor genome to copy when a live cell is created or overwritten. In evolution models, the usual procedure to choose an ancestor for offspring is to use either random (neutral) selection, a weighted probabilistic sampling or tournament selection based on the sequence-dependent fitness properties of the genomes. In the context of extending the GoL however, introducing random choices of ancestor introduces a possibly unnecessary lack of determinism into the model. If we consider firstly the neutral case, then it is important to observe that, in the GoL, birth occurs only for 3 live neighbours, and we observe that for all possible 3live-neighbour configurations there is a deterministically unique most different neighbour as seen in Supplementary Fig. 2. The same is true for all odd $s$ values, $\{1,3$, $5,7\}$. Only for the four non-zero even values $\{2,4,6,8\}$ of $s$ are there ambiguous configurations in which it is not possible to distinguish positionally between ancestors. In these cases, there are a number of options still to deterministically resolve matters: (i) not allow birth in these ambiguous cases, (ii) use the neighbourhoods of the neighbours to complete the choice of ancestor, (iii) complete birth with another e.g. GoL rule encoding sequence, (iv) perform recombination between the unresolved sequences, (v) distinguish sequences by fitness if different, or instead (vi) use random choice if other distinctions fail. Our implementation allows any of these options to be employed. The important point is that only a subset of configurations need resolution and even a deterministic algorithm which disallows transitions (as in (i)) in these cases is viable. Note that one version of (ii) of significance is to prefer ancestors which will not survive in the next time step, which brings us to the next point.

Movement vs. Copying. Whereas the indistinguishability of 1-states in binary CAs means that it is not possible to distinguish movement from death and rebirth, in this paper with genetic information attached to the live states, this is possible. We found that in the GoL and many related CA rules, in almost all cases in which a gene is chosen as an ancestor of a new live state, the gene does not survive at its previous location. This is significant because it means that two or more birth events are usually required for proliferation, and that single birth events can usually be interpreted as motion. We implemented an option for genelife in which we enforce death for parent genome cell states (in the cases that they are not anyway overwritten or not marked for survival by the CA rule table). This had little impact on the dynamics in the case examined. With this modification, the distinction between birth and movement is clear: movement occurs if an ancestor gives rise to one live cell at a neighbouring position and birth occurs if more than one live cell (and their copied genomes) arise from that ancestor. It would make a difference if mutation were deemed not to occur for transitions involving movement. Also, it might be appropriate to make the choice of an ancestor sensitive to the interpretation of movement $v s$ birth: e.g. to minimize the number of births needed to maintain the dynamics. For example, an isolated rod of three live states is a GoL oscillator between vertical and horizontal configurations. In the deterministic most different ancestor canonical assignment of ancestors from three live neighbours, the central gene is copied to two new sites so that (without mutation) the rod becomes genetically homogeneous in one step. This process is clearly a copy mechanism. On the other hand, in the 0-bit canonical assignment of ancestors, the two peripheral genes circulate anti-clockwise and this is more naturally understood as a 
process of motion and as such should be carried out without mutation. We postpone further investigation of the ramifications of distinctions between movement and copying for later work.

Additional Selection Mechanisms. Fundamentally, models of selection distinguish three modes of selection:

1. The neutral model, for which the deterministic position-dependent choice of ancestor outlined above, see also Electronic Supplementary Material, is an alternative to random choice.

2. Selection based on comparing fitness as a property of a single individual, independent of the presence of other individuals in the neighbourhood. This has the property of well-ordering all the genetic sequences (by fitness), with transitivity in comparisons ensured: i.e. $\mathrm{A}>\mathrm{B}$ and $\mathrm{B}>\mathrm{C}$ implies $\mathrm{A}>\mathrm{C}$. The focus is here on selection mechanisms that attribute an increasing cost to more prolific (less GoL-like) and more specific rule specification, fostering complex dynamics close to GoL. We do investigate the impact, for comparison, of a number of simple sequence-level selection pressures like selection favouring genetic sequences with more or less ones.

3. Selection based on a contest or tournament between individuals in which fitness depends on the other individual involved, there is no transitive ordering of genomes, and in population terms the fitness of a genome is population density dependent. Perhaps the best-known example of strongly not well-ordered fitness is in the scissors-paper-stone game $\mathrm{A}>\mathrm{B}>\mathrm{C}>\mathrm{A}$. We chose instead the four sequence classes (A, B, C, D) with selection $\mathrm{A}>\mathrm{D}>\mathrm{C}>\mathrm{A}$ as well as $\mathrm{D}>\mathrm{B}$ and $\mathrm{C}>\mathrm{B}$. This scheme is interesting in specifically supporting a multi-sequence coexistence making use of multiple spaceships.

Note that independently of these optional additional selection mechanisms on the choice of ancestor, the cooperative genetic coding of rules for LUT transitions means that there is also an underlying selection for sequences which can induce proliferation in their locality effectively through supporting birth or survival rules.

Rule Symmetries. For genetically dependent rules, a natural first family is the extended GoL symmetry class of $2^{18}$ semi-totalistic rules, distinguishing 18 states (2 central states times 9: the sum $s$ of live neighbours ranging from 0 to 8 ). The sparsity of such rules with properties near to the GoL led us to also consider the broader families of rules with lower symmetries, distinguishing up to 64 local neighbourhood states (the maximum for which a 64-bit genome can encode the rule). The full set of $512=2^{8+1}$ distinguished, local states ( 9 cells), giving rise to $2^{512}$ possible rules, is too large to explore initially, especially with our restricted length genomes, and is physically less appealing because it does not take spatial symmetry into account. Between semitotalism and full asymmetry we identify three intermediate symmetries (cases 3-5 below). This leads to the following six cases, of which we implement and study the first five:

1. Semi-totalistic rules with $s=2,3$ (gene dynamics with fixed homogeneous rules) $(4 / 4)$

2. Semi-totalistic rules $s=1-8$ with LUTs for 1 -states determined by genes $(16 / 18)$ 
3. Quarter-totalistic rules $s=1-7$, se $=0-4$ with distinct corner and edge counts $(46 / 50)$

4. Octavian-rotation symmetric rules for $s=2-6$, crot $=0-9(64 / 68)$

5. Isotropic rules in 2D (4-rot'n, 4-refl'n) for $s=0-4$, crot $=0-12(64 / 102)$

6. Full set of rules without symmetry reductions (512).

The numbers in parentheses are the number of distinguished local configurations in the specified family of local CA rules: i.e. the number of bits required to specify the rules, for (chosen $s /$ all $s$ ). These configurations divide into the two equal-sized subsets, birth/movement and survival: The birth/movement rules involve copying/moving a genome from one of the neighbours. The survival rules for current state " 1 " define the other exceptions to the default rule which is next state " 0 ". For each value of $s$, we also introduce a control bit "overwrite" which determines whether birth/movement rules can overwrite an existing live cell or not. These are collected into a mask overwrite for the eight $s$ values $1-8$, with semi-totalistic control of this property (no $s=0$ overwrite).

We found that the semi-totalistic case is too coarse an encoding of CA rules, to allow significant genetic evolution of complex structures beyond the classic game of life. We investigate the more differentiated rules and find that they produce a range of interesting dynamics. When coupled to a genetic population, these differentiated rules rapidly evolve to proliferate unless very strongly constrained (by the birth-survival mask introduced above).

We preface this study in Sect. 2.1 with rules which, like the GoL rule, can only yield live cells for $s=2,3$ and for fixed rule departures without genetic determination. As we shall see, some of the interesting evolutionary phenomena revealed by Genelife are already captured by this simplest case. Naively, one would expect $2 \times 2=4$ distinguished states $(c, s)=(0$ or 1,2 or 3$)$ that can possibly lead to live states and hence $2^{4}=16$ different genetic extensions. We perform a survey of the additional choices available for coupling genetics with the dynamics in this first case and implement these in a unified computer program to explore the model properties.

\subsection{Semi-totalistic Rules Involving Only $s=2,3$}

Since the GoL starting from random compact patterns of live states almost certainly relaxes to a set of unconnected simple patterns or periodic structures, with new live states only being produced in a small number of contexts, it is not as it stands a good substrate for evolution. In contrast, the conventional approach generalizing the GoL [20], requires that the birth and survival rules form a single interval of neighbourhood sum values, with lower and upper limits in the sum variable $s$, restricting the possible rule-tables to a family with members specified by four integers $\mathrm{S}_{1} \mathrm{~S}_{\mathrm{u}} \mathrm{B}_{1} \mathrm{~B}_{\mathrm{u}}$. Most of these rules lead to strong proliferation of live states or their extinction, and in order to allow genetic encoding to deliver novel dynamics of interest it turns out to be important to further dissect the rule-tables in the vicinity of the GoL rule 2333.

Genetic modifications that reduce the number of configurations resulting in live states will further restrict the potential for ongoing evolution, which requires more active rules (more neighbourhood state configurations leading to live cells) than the GoL. The most parsimonious first choice is, as for the GoL, to continue to consider 
only rules with next live states for $s=2$ or 3 live nearest neighbours, i.e. distinguishing 4 neighbourhood states as candidates for a live next central state $c:(c, s) \in$ $\{(0,2),(0,3),(1,2),(1,3)\}$. Since there are 4 starting states and 2 predicted outcomes (live or not) for the next central cell state, there are $2^{4}$ such rules, corresponding to the subsets of the starting states that give rise to a live next state. The GoL rule has survival for $\mathrm{s}=2,3$ and birth for $\mathrm{s}=3$, corresponding to the subset $R_{G o L}=\{(0,3),(1,2),(1$, $3)\}$. Since we need more active rules, it is logical to begin with letting the genes control the missing birth rule $(0,2) \rightarrow 1$ for $s=2$. If the proliferation induced by this extra birth process should prove too strong, then one could counter this by removing one or more of the elements of $R_{G o L}$. This compensation could either be fixed or dependent on the genes. Since there are a number of intermediate and hybrid cases, we summarize the various options that we have investigated in Table 1.

In order to distinguish the genetic dependency from uniform changes in the rules, we split the survival and birth processes into two optionally executed stages, the first depending on the selective genetics $\left(\mathrm{S}_{\mathrm{g}}\right.$ and $\left.\mathrm{B}_{\mathrm{g}}\right)$ and the second genetically independent (i.e. enforced, $\mathrm{S}_{\mathrm{f}}$ and $\mathrm{B}_{\mathrm{f}}$ ), as shown in Table 1 . The first 8 binary options in Table 1 give rise to 144 different cases. For birth, all of the $2^{4}=16$ cases are different, in contrast with the case in survival, where there are only $3 \times 3=9$ cases. Even if birth is enforced, the genomes of the live neighbours may still have a vital impact on the future dynamics by determining which of them becomes the ancestor of the newly born genome. In addition to the distinction of birth and survival depending on the state of the central cell, there is another possibility opened up by the genetics which is not distinguished in the binary GoL: Instead of simply remaining alive, the genome of the next state may be overwritten by one of the neighbouring genomes according to a birth process. We label this binary option $\mathrm{O}_{2 / 3}$ as it may be allowed independently for $\mathrm{s}=2$, 3. This corresponds to the well-studied Moran model of population genetics [34]. There are thus 576 different genetic extension models, even before one considers details of the genetic dependency.

In the interests of further limiting and analysing the extent of rule departures from the GoL, we also record here for completeness two further binary options $\mathrm{N}_{\mathrm{r}}$ and $\mathrm{N}_{\mathrm{s}}$, which enforce GoL rules if respectively the previous transition rule was a non GoL rule or the current state was last produced by a non GoL transition. In near GoL simulations colouring cells by departures from the GoL rules in these two ways allows an assessment of both the potential and effective impact of the modified rules on the dynamics.

The first column of the table records the transition processes extending the GoL rules. Of the 4096 options opened up by this table, only 9/16 i.e. 2304 of them are distinct because only $3 / 4$ of the selective/enforced survival options are distinct. The remaining columns consist of an index number nr, the central cell state $\mathrm{c}$ to which the transition applies, the sum s of live neighbours, the transition notation where $\mathrm{S}$ and $\mathrm{B}$ stand for survival and birth and the subscripts $g$ and $\mathrm{f}$ for genetic and enforced. All twelve options except for 4 and 5 have been realized in the genelife software. 
Table 1. Binary options for the control of genelife restricted to 2 or 3 live neighbours.

\begin{tabular}{l|r|l|l|l}
\hline Transition & $\mathrm{Nr}$ & $c$ & $s$ & $S / B_{g / f}$ \\
\hline Selective genetic birth for 3 live neighbours & 0 & 0 & 3 & $\mathrm{~B}_{\mathrm{g} 3}$ \\
\hline Selective genetic birth for 2 live neighbours & 1 & 0 & 2 & $\mathrm{~B}_{\mathrm{g} 2}$ \\
\hline Enforce birth for 3 live neighbours & 2 & 0 & 3 & $\mathrm{~B}_{\mathrm{f} 3}$ \\
\hline Enforce birth for 2 live neighbours & 3 & 0 & 2 & $\mathrm{~B}_{\mathrm{f} 2}$ \\
\hline Selective survival for 3 live neighbours & 4 & 1 & 3 & $\mathrm{~S}_{\mathrm{g} 3}$ \\
\hline Selective survival for 2 live neighbours & 5 & 1 & 2 & $\mathrm{~S}_{\mathrm{g} 2}$ \\
\hline Enforce survival for 3 live neighbours & 6 & 1 & 3 & $\mathrm{~S}_{\mathrm{f} 3}$ \\
\hline Enforce survival for 2 live neighbours & 7 & 1 & 2 & $\mathrm{~S}_{\mathrm{f} 2}$ \\
\hline Birth overwrite for 3 live neighbours & 8 & 1 & 3 & $\mathrm{O}_{3}$ \\
\hline Birth overwrite for 2 live neighbours & 9 & 1 & 2 & $\mathrm{O}_{2}$ \\
\hline Enforce GoL rule if last rule non GoL & 10 & $0 / 1$ & $2 / 3$ & $\mathrm{~N}_{\mathrm{r}}$ \\
\hline Enforce GoL if last state change non GoL & 11 & $0 / 1$ & $2 / 3$ & $\mathrm{~N}_{\mathrm{s}}$ \\
\hline
\end{tabular}

There are still several decisions to be made associated with the choice of ancestor, even after the choices in Table 1 have been made. For example, even for neutral models one can support birth only if two genomes are the same or different making a neutral choice of which will be the ancestor for offspring between them if this criterion is fulfilled.

\subsection{Genetically Encoded Semi-totalistic Rules for $s=1-8$}

For the semi-totalistic case, if we exclude the special cases of spontaneous birth $\left(\mathrm{B}_{0}\right.$ i.e. birth for $s=0)$ and lone survival $\left(\mathrm{S}_{0}\right)$ then there are $8+8=16$ distinguished states that may be independently part of an active next state ruleset. In this case, genomes may specify any look up table (LUT) depending only on the central state $c$ and neighbour sum $s$ via two separate subsets of s-1 values for survival $(1 \rightarrow 1)$ and birth $(0 \rightarrow 1)$. The omission of $s=0$ birth is equivalent to there being no spontaneous generation of life without neighbouring information (as in the Pasteur experiment) but [20] uncovers interesting cases with $s=0$ survival allowed. For $s=1 . .8$, there are $2^{16}$ $\mathrm{CA}$ rulesets, and these may be encoded by a binary genome of length 16 with one bit per LUT entry. In this paper, we restrict our attention to genomes of maximal length 64, and often use the term genome to refer to this full sequence, reserving the term gene to refer to a specifier of part of the rule-table. We may also employ multiple bits $\left(n_{c}\right)$ to encode each LUT entry for an active rule, for example with only one of the possible $n_{c}$ gene patterns being active, then genomes of length $16 \times n_{c}$ are required. In this paper, we only consider this option for the semi-totalistic case, where there is sufficient length in the genome to allow the range of values $n_{c}=1,2$, or 4 .

An alternative modular encoding employs 4 bits per entry: 3 bits to encode the values of $s$ for which the next state is live, plus 1 bit for survival or birth. The standard GoL would require at least $3 \times 4=12$ bits to be specified, $0 x b 23$ in hexadecimal notation, so that 64-bit genomes such as 0xaaaaaaaa22221111 or any other combination of only the three digits 1, 2, a would encode the GoL. Longer genomes may 
contain the same entry repeatedly allowing for mutational error resistance, especially when the birth-survival mask makes a significant number of entries ineffective. For example, the sequence 0x0000000000000a21, encodes the GoL local rule if the birthsurvival mask is 0 for $\mathrm{s}=1$.

\subsection{Quarter-Totalistic Rules $s=1-8, s e=0-4$, Counting Corners/Edges (46)}

It is clear from geometry that of the 8 neighbour sites, the four corner sites are further away (distance $\sqrt{2}$ ) from the centre site than the edge centred sites (distance 1) on a unit square lattice. We count the number of live edge centred sites as se, then if distance is taken into account and neighbouring groupings otherwise ignored the state transitions of a quarter totalistic CA depend on the tuple $(s, s e)$ with the possible values of $s e$ ranging from $\max (0, \mathrm{~s}-4)$ to $\min (s, 4)$ with numbers of different configurations for $\mathrm{s}=0-8$ of $\{1,2,3,4,5,4,3,2,1\}$, in total 25 . If we exclude the configurations $\mathrm{s}=0$, 8 , for which this distinction plays no role compared with the semi-totalistic case, then there are 23 different neighbour configurations distinguished by this symmetry and a total of 46 different LUT entries for survival and birth. Only 1-bit direct encodings of LUT transitions are possible in a 64-bit genome, but modular encoding of individual rules is also allowed: using 6-bits per rule as a combination of (i) 4 bits to specify B/S and $s-1$ (ii) 2 bits to specify $s e$ in the range $0 . .3$. The value $(s, s e)=(4,4)$ is placed (and decoded) as an exception in one of the unreached bit combinations. At most ten such modules can be encoded on a single genome, sufficient to specify the GoL rules and many others.

Actually if we count the sum of the weighted distances, each $s$, se configuration is different, and so that this quarter-totalistic symmetry actually warrants consideration as a variant in the semi-totalistic family of rules which only depend on the sum of the live neighbours (in this case weighted sum).

\subsection{Octavian Symmetry Genetically Encoded Rules for $s=2-6$ (64)}

An alternative symmetry breaking of the semi-totalistic case involves 8-fold rotation symmetry in which distinctions between corner and edge states are ignored (the 8 neighbouring lattice positions being regarded as lying equally spaced on a ring). While it is not possible to do this simultaneously in 2D for all sites, one could consider this symmetry as being physical when the potential of direct contact to a neighbour is more important than the distance. In contrast with the semi-totalistic case we then distinguish the groupings of live cells around this ring, regarding all 8 rotations of the ring as symmetric. This simple octavian symmetry results in the following numbers of different patterns for each value of $s$ from $0-8\{1,1,4,7,10,7,4,1,1\}$. Since we are most interested in cases generalizing the $s=2,3 \mathrm{GoL}$ rules, and the cases $s=0,1,7,8$ can be deemed fundamentally of less interest, we concentrate on the central values of $s=2-6$ for which the 32 different configurations (up to octavian symmetry) allow 
possible 32 survival and 32 birth rules. This choice is expedient, allowing a direct onebit genetic encoding of each transition rule for a 1 at the next time step to fit in a 64-bit genome. An alternative hybrid modular encoding of rules uses a combination of (i) 5 bits to specify B/S, $s-1$ and which of two sets of 5 distinguished configurations is addressed and (ii) 5 bits as a mask specifying which of these 5 configurations is active. At most six such modules can be encoded on a single genome, sufficient to specify the GoL rules for example.

The symmetry class and hence LUT entry for any specific configuration of live neighbours can be found efficiently by defining a canonical minimum value over the eight possible rotations of an eight bit pattern, regarded as an eight bit integer, and mapping any configuration to its canonical minimum value to find its index in the LUT.

\subsection{Isotropic Genetically Encoded Rules for $s=0-4$ (64)}

With full 2D spatial symmetry (4 rotations and 4 reflections), the numbers of distinguished configurations for $s=0 . .8$ are $\{1,2,6,10,13,10,6,2,1\}$ with sum 51 , still many fewer than the full asymmetric distinguished numbers $C_{s}^{8},\{1,8,28,56,70,56$, $28,8,1\}$, with sum 256 . In this study, with our 64-bit genomes, we will only fully investigate the evolution of differentiated rules for the lower range $s=0 . .4$, with 32 distinguished configurations, using 1 bit each in the genome to specify survival and birth rules in the direct encoding. As in Sect. 2.4, an alternative hybrid modular encoding of rules uses this time a combination of (i) 6 bits to specify B/S, $s$ and which of two sets of 6 distinguished configurations is addressed and (ii) 6 bits as a mask specifying which of these 6 configurations is active. At most five such modules can be encoded on a single genome, sufficient to specify the GoL rules for example.

\section{Analytical Tools for Spatial Genetic Computation}

We focus in this section on a defining a set of analysis tools that can be applied to analyse both the spatio-temporal and genetic evolution in genetic cellular automata models as well as the extent of natural computation taking place. Not only can the system be analysed at the current time in terms of spatial patterns for both the binary live/dead cell states and for genetically resolved patterns, but the spatio-temporal dynamics can also be studied in terms of the time-evolution of spatial patterns or of genes, the latter involving potentially both genealogies and spatial patterns. We commence with simple local classification tools and then extend them to more global ones.

\subsection{Spatial Visualization of Cell Array}

The first and most immediate set of tools for analysis involve classifications of local information on the cell array, using specifically graded or discrete colour combinations. 
Genomes. The black and white representation of empty and live cells in the array for binary cellular automata can be further differentiated by using a variety of colour schemes for genetic information: genotypic, phenotypic or ancestral, all pertaining to the genomes present locally, either at the current time or in relation to previous times.

Genotypic Hash Colouring. The genetic sequence space (currently binary of length 64) can be mapped to 24-bit colour on 3 8-bit channels (red, green, blue) by a pseudorandom hash function. This will ascribe a particular colour to any live cell based on the gene value at the cell, with empty cells being displayed as black. This colouring does not preserve the topology of Hamming distance in the sequence space, but typically separates nearest neighbours strongly, so that even single point mutations can be readily detected.

Phenotypic Colouring. A phenotype in evolutionary biology is the set of observable traits or properties of an individual (which may be determined in complex ways from a genetic sequence or genotype) that contributes to its fitness (survival of its inheritable information to the next generation). For example, if the choice of ancestor in a proliferation rule is based on the number of $1 \mathrm{~s}$ in its binary genetic sequence, then a graded colour scale which changes from blue to red as the number of $1 \mathrm{~s}$ increases from 0 to $v$ is an example of a phenotypic colouring. For genes encoding local LUTs, the number of coded entries or the number of rules for survival or birth may be regarded as phenotypic indicators and coloured accordingly.

Ancestral Colouring. Since the topology of the hypercube is high-dimensional, it is not possible to embed this smoothly in colour space, to allow neighbouring sequences to have neighbouring colours, although a self-organizing feature map could in principle be employed to approximate this. Instead, we may group related sequences by common ancestor, choosing the hash colour (see above) of the ancestor to label cells. Two types of ancestor are employed in this work, both at a selectable number of genetic changes back in the past, producing two families of colourings. The novel ancestry approach steps directly from a gene to the first entry of the gene's ancestor in the population history. In contrast, the clonal ancestor steps to the immediate clonal ancestor of a clone. A clone is a set of identical sequences produced by a connected sequence of birth events. These spatial analysers of genetic relations are complemented below by the corresponding structural and temporal genealogies in the set of tools with temporal axes.

Dynamical Patterns. The genelife model exhibits spatiotemporal pattern formation, both in the sequence of spatial arrangements of live cells and in their genetic differences. The temporal sequence of these patterns can be analysed locally or globally in time and space. In order to support global temporal analysis, all genes and all spatial patterns that are produced during a model simulation are recorded using hash tables. In order for spatial patterns beyond nearest neighbours (such as spaceships) to be recorded one needs a mechanism for segmenting the lattice. In this paper, we employ a rapid online connected component labelling algorithm to distinguish spatial patterns. 
Transition Class Colouring. Transitions between states in genelife can firstly be classified as events of four types: death, survival, movement, birth. Combined events involving both death and movement or birth are possible if overwriting is permitted in the model. Furthermore, birth processes may be classified as clonal (exact inheritance) or with mutation. All these classes of events can further be divided into classes for different neighbourhood configurations depending on the symmetry of the model: using $s$ for semi-totalistic symmetry, $(s, s e)$ for quarter totalistic etc. Alternatively, transitions can be classified as conforming to the GoL rule for that configuration.

Extended Neighbourhood Period-One Spaceships. Fast parallel bit mapping macros are employed to map the $7 \times 7$ array of up to third nearest (Moore) neighbouring live/empty states into a single 64-bit integer for local processing. These packed integers are then compared efficiently with those at the previous time step of their eight lattice neighbours (i.e. comparing overlapping offset-1 patterns in the directions $\mathrm{NW}, \mathrm{N}, \mathrm{NE}$, $\mathrm{E}, \mathrm{SE}, \mathrm{S}, \mathrm{SW}, \mathrm{W}$ ) to detect preserved or nearly preserved patterns (period 1 spaceships). The cells are coloured by the direction of travel with brightness depending on the quality of pattern preservation.

Connected Components. Rapid connected component labelling algorithms, as reviewed in [35], can be employed to distinguish spatial patterns at every step of the simulation. We implemented a modified linear two-scan equivalent-label resolving algorithm with 8-neighbourhood, employing rank union-find to resolve labels, the Suzuki decision tree [36] (Wu's enhancement [37] did not result in significant efficiency gains) and periodic BCs. The algorithm was adapted to work with both binary images (live/empty state) and with genetically differentiated images in which connected components must consist of the same genome or more permissively genomes with a common ancestor at some specified level or time (ancestors may be either global or clonal, see Sect. 3.4).

The connected components can then be extracted efficiently as quadtrees [38], i.e. as a 4-tree of sub-squares, storing all sub-patterns encountered in the hierarchical construction of a quadtree in a pattern hash table (using the same software hashtable.h as the genomes). Small patterns $(8 \times 8$ pixels or smaller $)$ are keyed directly by their 64 bit binary patterns while larger patterns are keyed by combining the four 64-bit addresses of their four square sub-image patterns $\{\mathrm{NW}, \mathrm{NE}, \mathrm{SE}, \mathrm{SW}\}$ to a single 64-bit hash key with custom code minimizing collisions. This quadtree encoding is similar to but with a different handoff between large and small patterns to that employed in hashlife [39] for Gosper's algorithm [40] and our code development was aided by that implementation.

Although it would be an improvement for pattern recognition, we did not invest in reducing patterns to single representatives of their symmetry class (e.g. by rotation or reflection in the case of the isotropic rule sets of Sect. 2.5). The connected component patterns typically separated into a set of small frequently occurring patterns and larger patterns only found once in the simulation. This was more pronounced for the binary patterns. Larger connected components with the same genome are rarer. Since all 
patterns found in the simulation are saved in the hash tables, it is a single lookup to ascertain whether a pattern is novel, and to count reoccurrences of patterns. Thus, we enhanced the connected component labelling of different components by colour with an optional novelty filter, which darkens the colours of patterns that have already been encountered.

In order to track the information in spatial patterns over time, it is important to map the connected components at one time to those at the next time step. For unique assignment this is a linear assignment problem, and we initially used the LAPMOD algorithm [41] to find an optimal map between components. However, the more general problem of finding an (optimal incomplete) alignment matching components with weights determined by the pixel overlap between patterns, can be addressed as a maximal flow problem on bipartite graphs. A useful source of information on these algorithms is Tim Roughgarden's Stanford lectures (CS261). We modified the Hopcroft-Karp algorithm for maximal flow, with worst case execution time $O(|E| \sqrt{|V|})$ in terms of the number of edges $|E|$ and vertices $|V|$ in the bipartite graph, based on the implementation of Gupta [42]. Typically, connected components at one time step overlap with one or a few connected components at the next time step, so that a genealogy of connected components could be studied. However, we do not present this in the current paper. Instead, we use the mappings between connected components to provide continuity in tracking patterns over time and in colouring the cellular automata.

Displacement Genealogies. The above two methods are capable of labelling simple spaceships effectively which do not execute a complex periodic set of shape transformations. Here we introduce a novel dynamical pattern analysis tool, made possible by the genetic inheritance in the genelife model. We introduce a short-term dynamical memory packed into a 64-bit integer, in keeping with our general strategy of efficient machine integer-level processing. The novel feature is that this dynamical memory follows the genealogical inheritance path of live cell survival and ancestry, rather than sampling an expanding set of neighbour configurations. To be precise, any live cell at time t persists either by a survival or birth rule, and so at each time step and for each live cell, in the case of birth we first copy the integer dynamical memory (initially zero and termed golr) of the ancestor, and then in both cases we push (left shift and bitwise or) 4 bits of information to it: the four bits are a birth/survival bit and for birth a 3-bit displacement index $0 . .7$ for $\{\mathrm{NW}, \mathrm{N}, \mathrm{NE}, \mathrm{E}, \mathrm{SE}, \mathrm{S}, \mathrm{SW}, \mathrm{W}\}$ of the ancestor and for survival the 3-bit value $s$-1 mod 8 . This short-term dynamical memory ( $g$ olr) value thus records the ancestral displacement and survival configuration dynamics for the last 16 time-steps.

The displacement genealogy can then be used to identify various dynamical structures in the current CA. It has the advantage, compared with other dynamical records, of tracking the dynamics associated with the transmission of the live cell genetic information. For display we can further process this record by analysing the memory for periodicities and near periodicities, by calculating the minimal mismatch 
4-bit byte shift (period) when comparing the dynamical memory with its shifted value and calculating the mean displacement of the dynamical memory for its optimal period. We use a colour representation to display important features of this dynamical memory, in which brightness corresponds to quality of match, one channel (blue) corresponds to the optimal period (1-15 or 0 if none above a matching threshold) and one channel (red) corresponds to the mean displacement at the optimal period.

We note that capturing the essential dynamics of information processing in dynamical systems has been a major challenge, with the two major approaches of information transfer entropy [22, 23, 43, 44] and $\varepsilon$-machines [25, 26, 28, 45, 46] proving increasingly computationally intractable as one moves from $1 \mathrm{D}$ CAs to $2 \mathrm{D}$ systems for larger periodicities. Facing these difficulties, neural networks have been used to extract statistically significant dynamical features (epsilon networks) in 2D [47].

\subsection{Global Time Evolution of Genomes, Clones and Patterns}

The global temporal evolution of the model can be captured by keeping track of the populations of all the genomes and patterns that occur. Although for natural systems a daunting proposition, it turns out that this is possible in the current framework using appropriate hash tables and segregation of patterns. For genomes we record each new genome that occurs in a simulation in a hash table. We use the efficient and selfcontained C-package hashtable.h [48] with 64-bit hash keys: for genomes these are the genomes directly, for clones we use the $x, y$ and $t$ coordinates of the birth place and time of the clone and for spatial patterns we use a 64-bit quadtree hash code of the root of the tree or the patterns directly as hash keys for $8 \times 8$ bit patterns or smaller. See Sect. 3.1 for a description of the binary, genomic and ancestrally discriminated connected component decompositions. Note that these patterns are not aggregated corresponding to the symmetry of the transition rules in the model: although this would have been a further useful condensation.

In a single pass through the current state, we can count the populations of extant genomes and clones and once appropriately processed for connected components, we can assemble populations for the spatial patterns too, distinguished by binary state, genome or common ancestor. We identify the traces over time of these populations by an appropriate colour label corresponding to the hash key.

Population Scaling. In order to provide a robust visualization of populations of different magnitudes, we introduce a saturation scaling approach which is gleaned from the Michaelis-Menten biochemical kinetics of enzymatic reactions. The following rescaling of populations $N_{i}^{t}$ using the mid-point parameter $N_{m}$ ( $c f$. Michaelis constant)

$$
N_{i}^{\prime t}=N_{i}^{t} \frac{N_{m}}{\left(N_{i}^{t}+N_{m}\right)}
$$

has the value $N_{m} / 2$ when $\mathrm{N}=N_{m}$, approximates to $N_{i}^{t}$ at low values and saturates at $N_{m}$ for high values of $N_{i}^{t}$. We also use this rescaling for activities in Sect. 3.2. 


\subsection{Evolutionary Activity}

Evolutionary activity is a statistic designed to measure when components of an evolving population are persistent by virtue of their contributions to members of a population $[49,50]$. The choice of component defines what aspect the statistics track; we will consider two examples, genomes and spatial patterns.

A component labelled by $i$ has activity at time $t$ given by

$$
A_{i}^{t}=\sum_{t^{\prime}=0}^{t} \mathrm{C}_{i}^{t}=A_{i}^{t-1}+\mathrm{C}_{i}^{t}
$$

where $C_{i}^{t}$ is a count of the component's presence at time $t$. We will refer to live activity as the activity of only the components that exist at the current time,

$$
L_{i}^{t}=A_{i}^{t} \theta\left(\mathbf{C}_{i}^{t}\right)
$$

where $\theta(x)=1$ if $x>0$, and 0 otherwise. Even neutral evolution produces components that can persist for some time, and there are ways to construct neutral models to adjust activity measurement to signal only activity beyond neutral [50-52]. We will not require this in the present work, the main reason being that the case where all genes have no effect reduces to the GoL, which asymptotically produces no new activity because its dynamics relaxes to a field of fixed and low-periodicity local patterns.

Activities of genes or spatial patterns are stored in the relevant hash tables and updated efficiently using the recursion above, using the linear dependence of $\mathrm{C}_{i}^{t}$ on individual components to update only those component contributions that change in the current state.

From the component activities, we may form the time-dependent distribution of activity, for all activity and live activity,

$$
P^{t}(A)=\sum_{i} \delta\left(A-A_{i}^{t}\right) \text { and } P^{t}(L)=\sum_{i} \delta\left(L-L_{i}^{t}\right)
$$

We will find it convenient to view activity in two ways, examples will be seen below, as a superposition of activity waves (one wave for each $i$ ), and as an evolution of the activity distribution, represented by the time trace of quantiles for each $P^{t}(A)$ (or $P^{t}(L)$ ). For visualizing the individual waves, we scale them as in Eq. (1) to see longlived, high activity waves together with newly produced low-activity waves. Typical population and activity traces of genomes are shown in Fig. 2 and the probability distribution of activities is characterized in Fig. 3.

Gene Activities and Populations. When we consider components to be genes, we may label components with $g$ (instead of $i$ ), and $C_{g}^{t}$ is simply the number of genomes $g$ present at time $t$ (Figs. 2 and 3). 

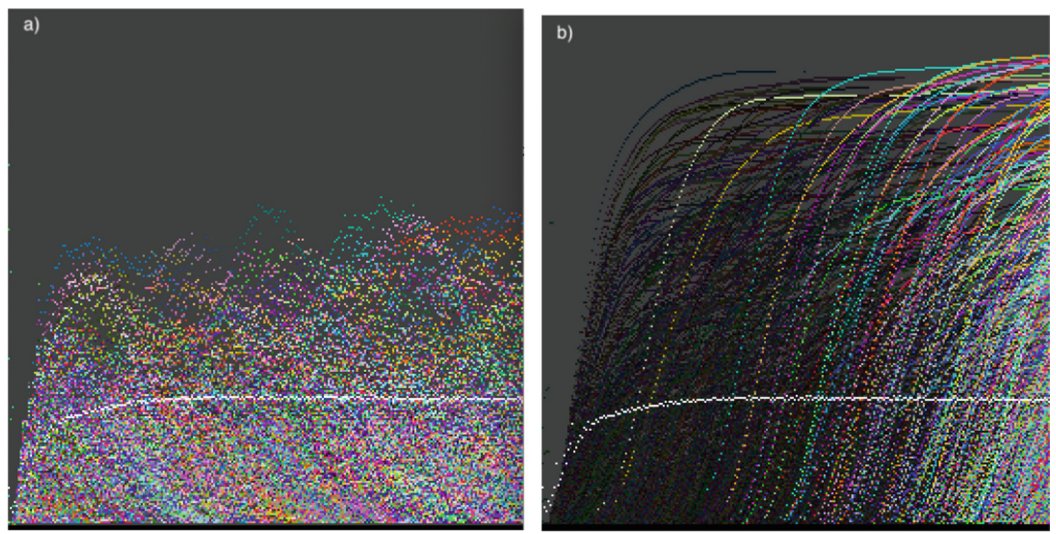

Fig. 2. Temporal evolution traces: (a) Population statistics of genomes $P_{g}^{t}$ and (b) Activity waves $A_{g}^{t}$ for $t=0$ to 128 . Populations, $P_{g}^{t}$, and live activity waves, $L_{g}^{t}$, are coloured with different shades of grey corresponding to different genomes $g$ and activity waves for genomes that are extinct are shown in black. The global density is in white (on scale 0 to 1 , bottom to top).
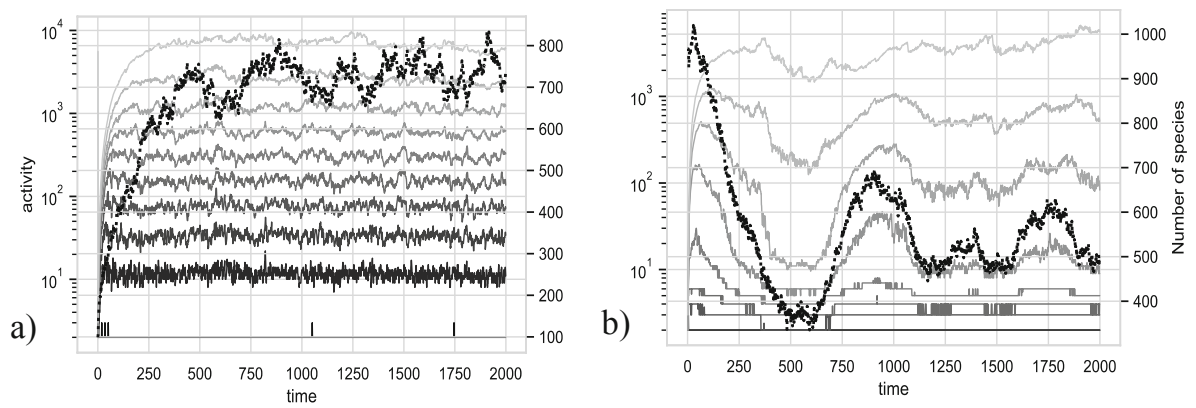

Fig. 3. Temporal evolution of deciles of the live activity distribution $P^{t}(L)$, for two examples of evolutionary dynamics, with different meta-parameters. The number of genetic species is shown with a dark dotted line. In (a) we see $P^{t}(L)$ converge to a rather stable distribution. Stable values of lower deciles imply that new genes are constantly being produced and are persisting in the population. In (b) we see a collapse of the genetic population for $\mathrm{t}<500$, followed by a recovery; persistence of new genomes in the following recovery is seen in a burst of new activity waves, reflected in the increase of low- and mid-level deciles from $t=700$ to $t=1000$.

Spatial Pattern Activities and Populations. As described above, connected spatial components may be rapidly detected for each time step. This enables the measurement of spatial pattern activity, which tells us whether there is an ongoing production of novel spatial patterns as a result of genelife evolution. The global record of spatial patterns can be traced via populations or activities (as shown in Fig. 4). 

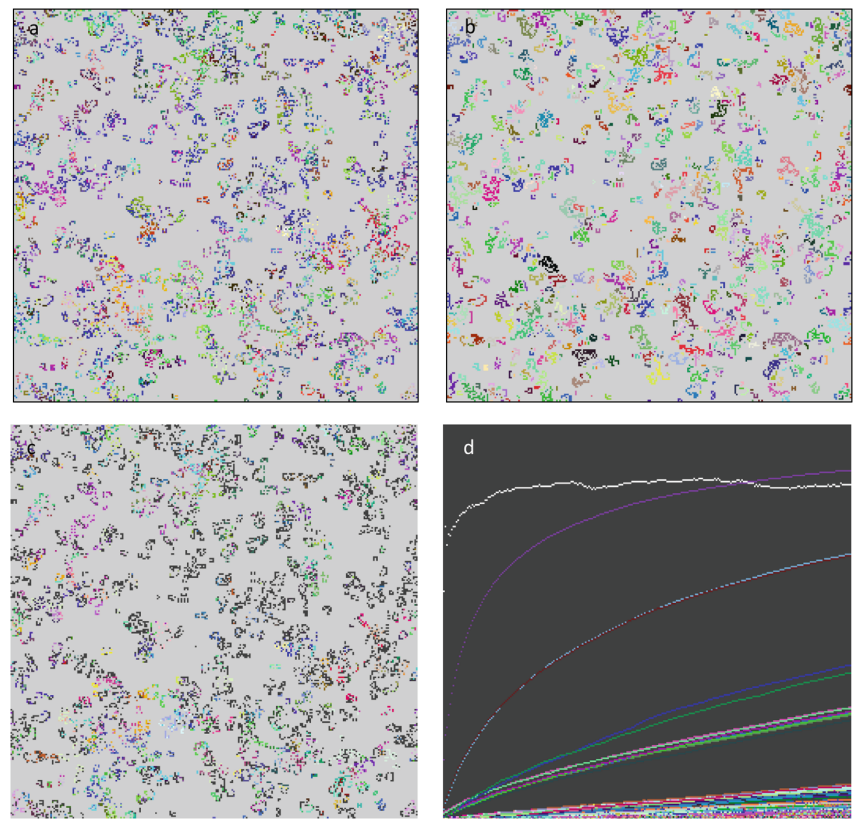

Fig. 4. Spatial pattern analysis and connection with genealogies. (a) The pattern of live cells after $\mathrm{t}=256$, coloured by genome identity; (b) The corresponding pattern of connected components, coloured by connected component identity - note that the connected components are often comprised of more than one genome; (c) Patterns coloured by common ancestor of clones at depth 2: The dark clones have root common ancestor, this is common at the early time shown; (d) Activity waves of connected components, each wave corresponding to a different connected component.

\subsection{Genealogies: Global and Clonal}

The study of inheritance in evolution has included genealogies and their statistics as an essential component in reconstructing the past, most recently and successfully in the form of coalescence theory [53-55], describing the statistics of the number of generations back to a common ancestor, applied to simple models of evolution [56] and the construction of evolutionary trees, the quality of which has been evaluated with statistical geometry [57]. Limited samples of sequences from current populations can be used to construct coalescence statistics and evolutionary trees. In genelife, inheritance is based on asexual reproduction, which simplifies genealogies since with a single parent the number of ancestors for an individual does not grow exponentially backwards in time. This allows a more complete record of ancestry to be recorded and updated at every timestep and the use of fast genealogical reconstruction to display the evolutionary development of a complete simulation.

Rather than consider every birth step, the majority of which do not introduce sequence changes (for the typically low mutation rates $\mathrm{ca} \cdot 10^{-2}$ to $10^{-3}$ appropriate for evolving short genomes of length 64 employed here), we introduce two abbreviations of complete genealogies, both of which only record genetic changes. The first and most 
extreme abbreviation is the first ancestor (global) genealogy, recording in the hash table entry for each genome encountered in the simulation both the time at which it first appears and the genome of its ancestor. Apart from initialization, or possibly a random influx of standard genes such as a GoL-encoding gene, which is given a special root sequence as ancestor, every new genome arises by mutation from an existing gene, its ancestor. Genealogical reconstruction is then straightforward, tracing through the hash table from first ancestor to first ancestor and noting also the time at which each ancestor first appears.

The second type of abbreviated genealogy we use is clonal, also tracing only genealogical inheritance steps that involve mutation, but tracing the genealogy for every clonal population of a genome (a clone is the identical progeny of each new mutant arising in the population). Each genome may appear multiple times in separate spatially or temporally separated clones in the population. Clones can be identified uniquely by the time and space coordinates of the cell in which the mutant genome of the clone is produced. This so called clonal birth-id may be used as a 64-bit hash key for storing all clones that occur in a simulation: each hash table entry records the ancestral clone from which it was produced and the genome of the clone as well as other statistics like the size of the clone and its evolutionary activity.

Visualization. In order to visualize efficiently the evolutionary trees resulting from these genealogies we use the same $N \times N$ array (matrix) of colour entries used to display the spatial pattern of the genelife CA. Along the horizontal axis of the array, each column is devoted to an extant genome or clone in the current population, choosing the $N$ most populated ones if there are more of them than can be displayed, and the time at which ancestors arise is displayed along the vertical axis, using discrete steps of length $t_{T} / N$, where $t_{T}$ is the total simulation time. In a single trace back of ancestors for the extant genomes or clones, we place the hash colour-id of the genome for each ancestor in the row corresponding to the time at which the ancestor was produced. If multiple ancestors occur in the same discrete time interval, the genome of the oldest is displayed (i.e. overwritten in the matrix). Figure 8 shows examples of first ancestor (global) genealogical trees which can be displayed in real time during a simulation. Colour and video material for these genealogies is available in the online supplementary information. In addition to the temporal vertical axis, Fig. 8 also shows genealogical depth plots in which the vertical axis shows simply the number of ancestral mutation steps, instead of their duration.

\subsection{Simulation and Analysis Software}

A systematic interactive simulation and analysis tool was written primarily in $\mathrm{C}$ (over 7000 lines of custom code, with additional use of 700 lines of hashtable.h code [48]) and with a custom python front end (using numpy, matplotlib, pySDL2) for graphics, simulation control and analysis ( $c a .2000$ lines of code), with Jupyter notebooks documenting standard usage and recording results. The software is written to take advantage of parallel bit processing in long integers but does not yet take advantage of GPUs or other parallel processing architectures, nor the special acceleration provided 
for example by Gosper's algorithm [40]. Emphasis was on a flexible generic platform supporting online analysis tools with global recording of novel structures found during the simulation. The software is available on github at http://github.com/js1200/genelife.

\section{Results}

In this section we present some evidence for several key findings revealed by application of the analysis tools to the simulation platform genelife, which invests the computational universe of CAs with the capabilities of evolution. Only an initial sample of the kind of new insights and results afforded by genelife can be presented in this paper. We first demonstrate the purely deterministic evolution, with ongoing innovation of patterns, exhibited by the model in the absence of mutation. We then show how increasingly fine dissections of the transition between subcritical and supercritical proliferation can be achieved using the successive symmetry breakings in Sect. 2. We demonstrate how ongoing evolution is revealed by the tools of activity statistics and genealogies and extend this analysis to the domain of spatial patterns. We leave the exploration of the information potential in initial patterns for controlling computation to future work. Instead, in the final section we explore one of the novel possibilities for characterizing the natural information occurring in evolving dynamical systems by introducing dynamical genealogies and exploring their ability to capture systematic computational motifs arising in the simulation. This provides an efficient genetic alternative for 2D CAs to epsilon machines [25], which were applied successfully to $1 \mathrm{D}$ CAs [29].

\subsection{Deterministic Dynamics of Evolution}

Case of No Mutation. The deterministic extension of the GoL through genelife is illustrated for the case of $\mathrm{s}=2,3$ with a non-well-ordered selection scheme as described in Sect. 2.1. The state of the $512 \times 512$ cell lattice after $c a .1000$ timesteps is depicted in Fig. 5, both for genomes and connected spatial components, along with several filters identifying travelling spaceship structures including the displacement genealogies of Sect. 3.1. Novel localized spatial patterns continue to arise as documented both by the activity statistics comparing times 500-1000 with times 950010000 , and by the novelty filtered components, a plot of number of live cells that are part of novel components over time (f). Using the global hash table for connected components, only the novel patterns are depicted in bright colours in Fig. 5c. The frequency of novel spatial patterns, occurring when spaceships collide giving birth to new spaceships, has not changed significantly even after 10x the simulation time. In contrast, only rarely are novel spaceships found after $\mathrm{t}=1000$. 


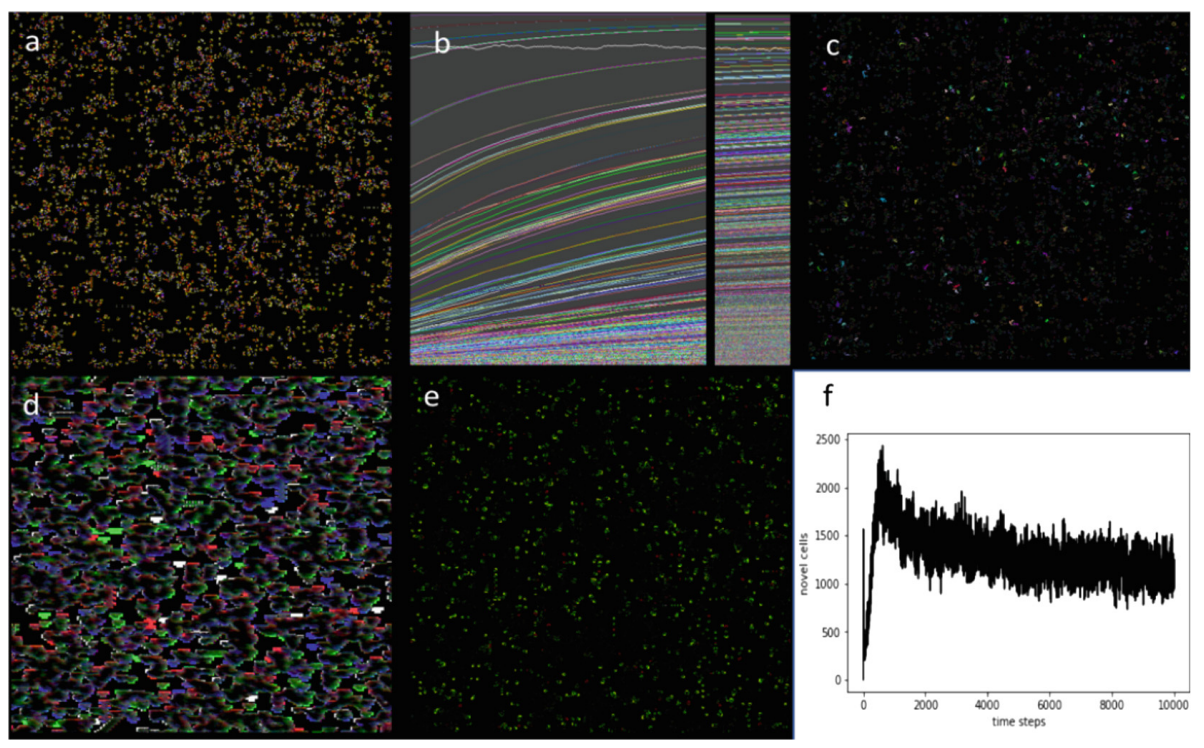

Fig. 5. Deterministic spatial evolution of ongoing novel patterns without mutation. (a-e) simulation status at $\mathrm{t}=1002$ (a) live cells coloured by genome (b) activity traces of connected patterns, left last 512 timesteps up to $t=1002$, right same for $t=10066$ (c) connected patterns with novelty filter darkening all non-novel patterns (d) fast spaceship detection using local $7 \times 7$ neighbourhood colouring cells moving in directions $\mathrm{N}, \mathrm{E}, \mathrm{S}, \mathrm{W}$ with red, green, blue and white (e) displacement genealogies identifying moving locally periodic automata with period 1 in green and longer periods (rare) in bluer tones, static structures in dull red. (f) number of live cells in novel components. (Color figure online)

It is clear even from this simple example, which could be reproduced in all of the symmetry breaking extensions in Sect. 2, that allowing different species into the GoL enriches the dynamics - from generic relaxation to very simple static or low period structures with rare gliders from random initial conditions, to relaxation to a robust and diverse population of spaceships that continue to repopulate the space and produce novel patterns at much longer times than are typical for the relaxation of the GoL.

\subsection{Symmetry Breaking from Semi-totalistic to Isotropic}

As discussed in Sects. 1 and 2, the GoL decays too strongly for almost all initial conditions to support a spontaneous supply of patterns that could act as a substrate for evolution. The capability of genetically modified local rule tables to modulate the dynamics near to the GoL rules is limited by the number of variants with proliferation rates near to the threshold between proliferation to fill the lattice and decay to rare quiescent local periodic structures as in the GoL. This depends on the spatial symmetry of the rules. For example, the semi-totalistic rules are sub-critical in the GoL for S23B3 but supercritical for S_B23 so that with s = 2 or 3 rules no closer approach to criticality is achievable. As an example of the novel capabilities introduced by symmetry 
breaking, as discussed in Sects. 2.2, 2.3, 2.4 and 2.5, in Fig. 6 we examine the ability of the symmetry broken S_B23 rule sets to evolve closer to the critical threshold for proliferation. Yet finer differentiation is possible by including combinations with differentiated survival, and exhaustive exploration of this threshold must await a study dedicated only to this point, since there are $2^{60}$ isotropic combinations to investigate just for the three numbers of live neighbour rules $\mathrm{s}=2,3,4$.
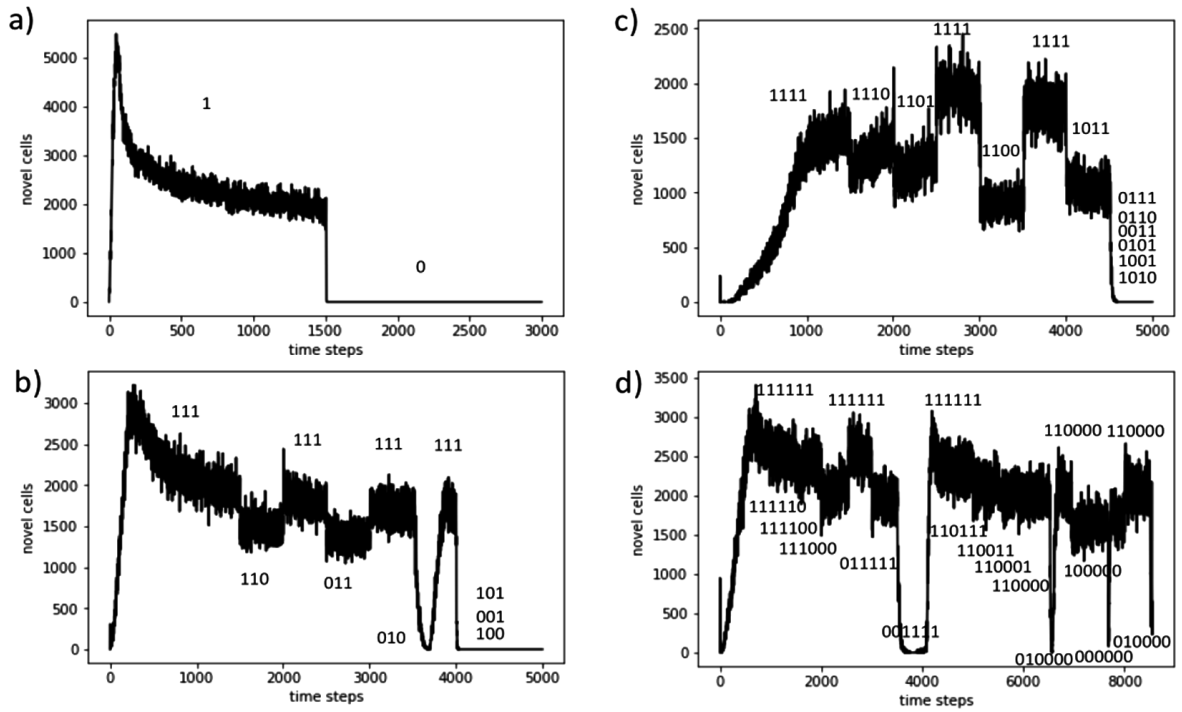

Fig. 6. Symmetry breaking of $s=2$ rules near to proliferation threshold. The number of live cells that are part of novel connected components is shown for the four symmetry classes in Sects. 2.2-5 (a) semi-totalistic, (b) quarter-totalistic, (c) octavian and (d) isotropic. The simulations were run for no survival but with overwrite with the entire $\mathrm{s}=3$ rule sets allowed, but only the masked members of the $\mathrm{s}=2$ rule (as shown in the text labels) were allowed: these values changed as shown during each simulation run. The genome choice was neutral but different genomes were required for birth (mode 5).

\subsection{Activities and Genealogies Show Ongoing Evolutionary Process}

Evolutionary activity has long been a signal of ongoing evolution; if a population continuously produces new genetic variants that succeed in surviving and propagating in the population, the population continues to evolve. We have seen, in the case of deterministic evolution discussed in Sect. 4.1, that even when the genetic population is constrained to have no innovation (suppressing random mutation), evolutionary activity of spatial patterns reveals that evolution can proceed robustly nonetheless.

Evolution within the more simply defined rule spaces tends to produce a certain kind of open-endedness, with ongoing production of new genetic material that is successfully absorbed into the population. However, the dynamics appears to be quite similar, notwithstanding the new genes being absorbed into the population. This is a 
signal that these new genes are drawn from a genetic subspace that is neutral with respect to the dynamics, and hence the ongoing production of genetic activity does not produce new dynamical functionality.
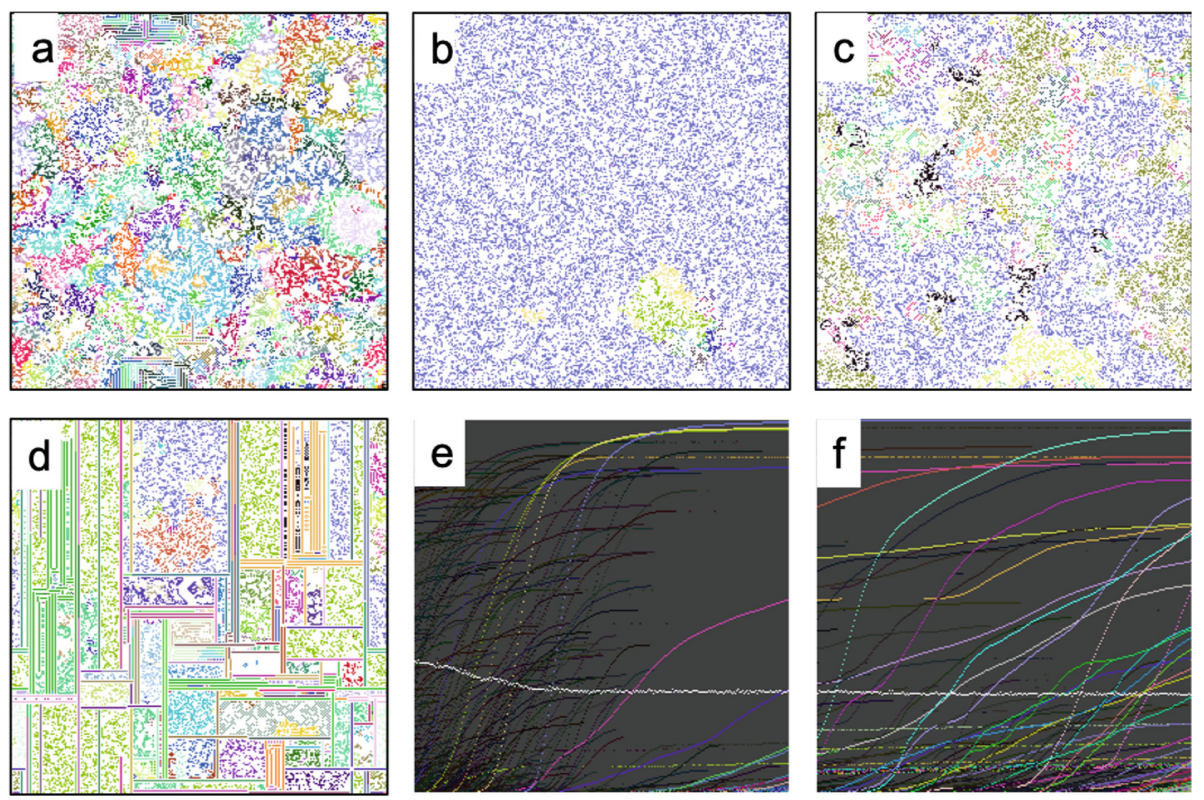

Fig. 7. Complex evolution with quarter-totalistic rules (see Sect. 2.3). Panes (a)-(c) describe a sequence proceeding from one dynamical phase (a) early expanding blooms (b) $t \approx 550$ near collapse of the population to one dominant genome (c) $t=8000$ successful invasion of new genomes that evolve and persist, with dynamics of competing domains that are more stable than the initial bloom dynamics. Pane (d) illustrates evolution in a similar universe of quarter-totalistic rules, producing an ecology that asymptotically evolves domains, with robust evolving dynamics within each domain. Temporal evolutionary activity waves $A_{g}^{t}$ are traced in (e) and (f) at early and later times. Live activity waves, $L_{g}^{t}$, are coloured with different colours (shades of grey) corresponding to different genomes $g$ and activity waves for genomes that are extinct are shown in black. The global density is shown in white (on scale 0 to 1 , bottom to top).

This limitation on evolvability was in fact a motivation to consider more complex rule spaces obtained by breaking symmetries of the rule space as described in Sects. 2.3, 2.3, 2.4 and 2.5. Figure 7 illustrates a complex evolutionary sequence from the quarter totalistic rules of Sect. 2.3. In the evolutionary sequence illustrated, we see a 'major transition' in the evolution of the population, at about $t=550$, when the population collapses toward a single dominant genome, but then is re-infected with genes that succeed in propagating and persisting for continued ongoing evolution. 
The genealogies for the evolutionary sequence illustrated in Fig. 7 are shown in Fig. 8. The collapse drastically reduces the number of genomes present in the population, but recovery enables rebuilding of a genetically rich population. A movie showing the simulation dynamics is included as Supp. Fig. 6.
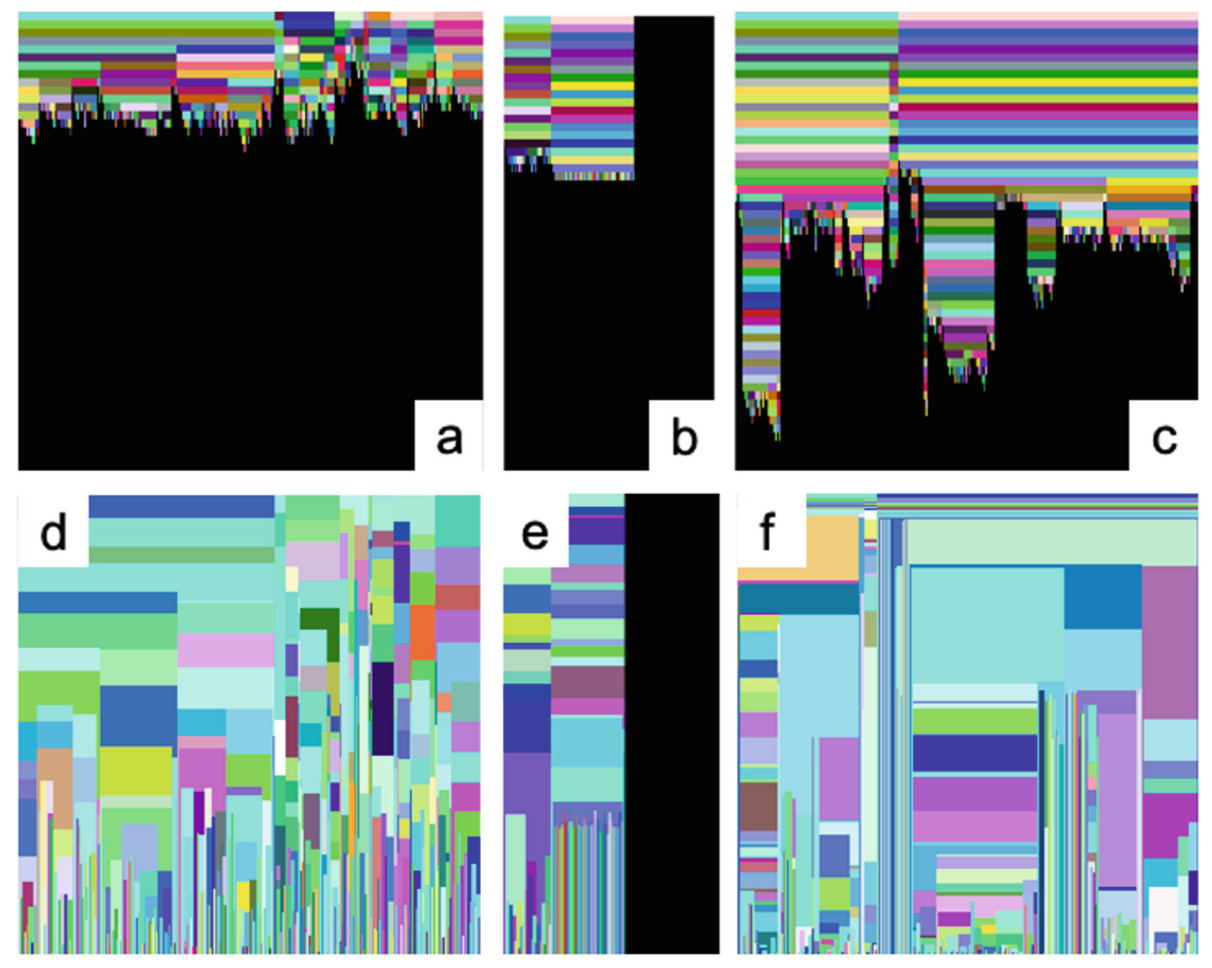

Fig. 8. Genealogies for the three phases of evolution in Fig. 7(a), (b), and (c), at the same time steps as those figures. These genealogies are colour coded by genome and successive generations occupy rows from the top (earliest time) to the present, coalescing backwards in time. The corresponding time-resolved genealogies are shown in (d), (e), and (f), with time between gene shifts represented as the vertical extent of each coloured patch representing a genome.

Evolutionary activity, both genealogical activity and spatial pattern activity, reveal the evolutionary dynamics to be open-ended. Activity associated with the evolution of new functionality remains, however, elusive. We see what may be interpreted as major transitions $[19,58]$ in the collapse and recovery illustrated in Fig. 7.

\subsection{Displacement Genealogies, Computation and Selection}

Computation is traditionally accomplished in the Game of Life by transmission of information using gliders, and locally oscillating and translating configurations. In 
other CAs, information is transmitted via traveling boundaries between phases [25]. Genelife has the additional feature that besides spatial patterns causing information transfer, genetic information is also transferred as a result of dynamics. In order to capture the genetic information transfer, we construct displacement genealogy histories, as in Sect. 3.1: every live cell has a short history of where the genome's information has come from in the past, including all spatial translations during this history. Such displacement genealogies can be further reduced to state transition diagrams and their statistics analysed as shown in Fig. 9.
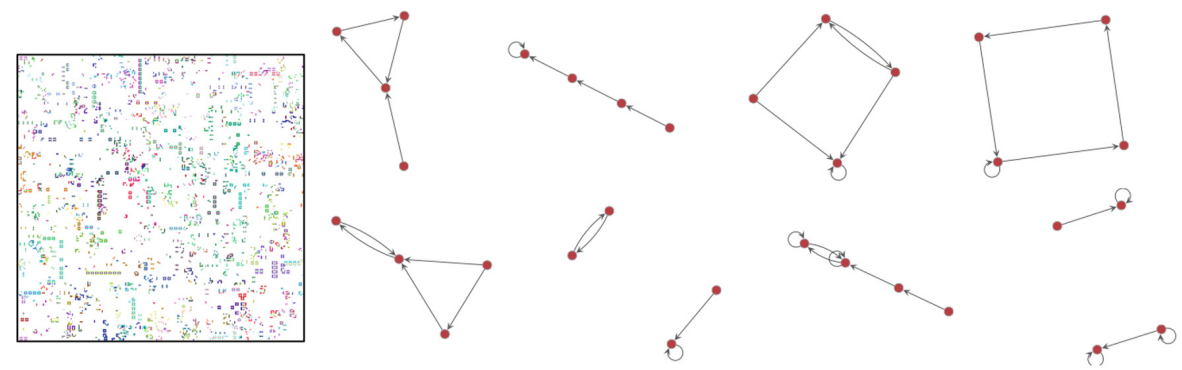

Fig. 9. Evolution in a universe of semi-totalistic rules described in Sect. 2.2 produces a glider rich population, continually evolving (shown on the left). At time $t=500$, each live cell was polled for its history of the last sixteen timesteps of displacement genealogy, to construct a state transition diagram. These state transition diagrams were collected across all live cells, and the eight most common are shown on the right.

Displacement genealogies may be used to capture computational state transitions performed by the spatial dynamics of the genomes. This is a simple, but concrete method of empirically reducing the dynamics to computational state transitions, involving a 1D thread of information following the path of genetic transmission, and while not involving as much computation as with the $\varepsilon$-machine construction [25, 47] has advantages in $2 \mathrm{D}$ where the amount of information needing to be digested for the construction of $\varepsilon$-machines becomes prohibitive. It is well applicable for dynamics that produce many interacting periodic structures (gliders and spaceships). If the dynamics are too dense, spaceships travel only very short distances before collision and their displacement genealogies proliferate to the point that statistics are difficult to collect. At higher densities other regular structures of spatial genetic transmission may arise that can then also be captured by displacement genealogies. The local displacement genealogies can also be analysed for most prominent period and mean displacement at this period and coloured accordingly; an example is shown in Fig. 10.

\subsection{Selection Based on Displacement Memory}

As an extension of the model, without changing the relationship between genomes and rule tables, the choice of ancestors can be made dependent on the current displacement genealogy (in the same cell as genome). We made this step in order to examine whether 
an autonomous selection for genomes that modify the rules in such a way as to give rise to desirable local state genomic state machines (as depicted in Fig. 9) can occur. To this end we introduced a set of additional selection options that select for precise periodicity, for large or small periods, for large or diagonal displacements. Interestingly, rich repertoires of spaceships, as in the non-well-ordered selection model case of Sect. 4.1, were found generically in such models, demonstrating that local selection for dynamical traits is effective in genomic evolution, despite the indirect nature of the selection feedback (with multiple genomes determining local rules together). An example of such a simulation is shown in Fig. 10.
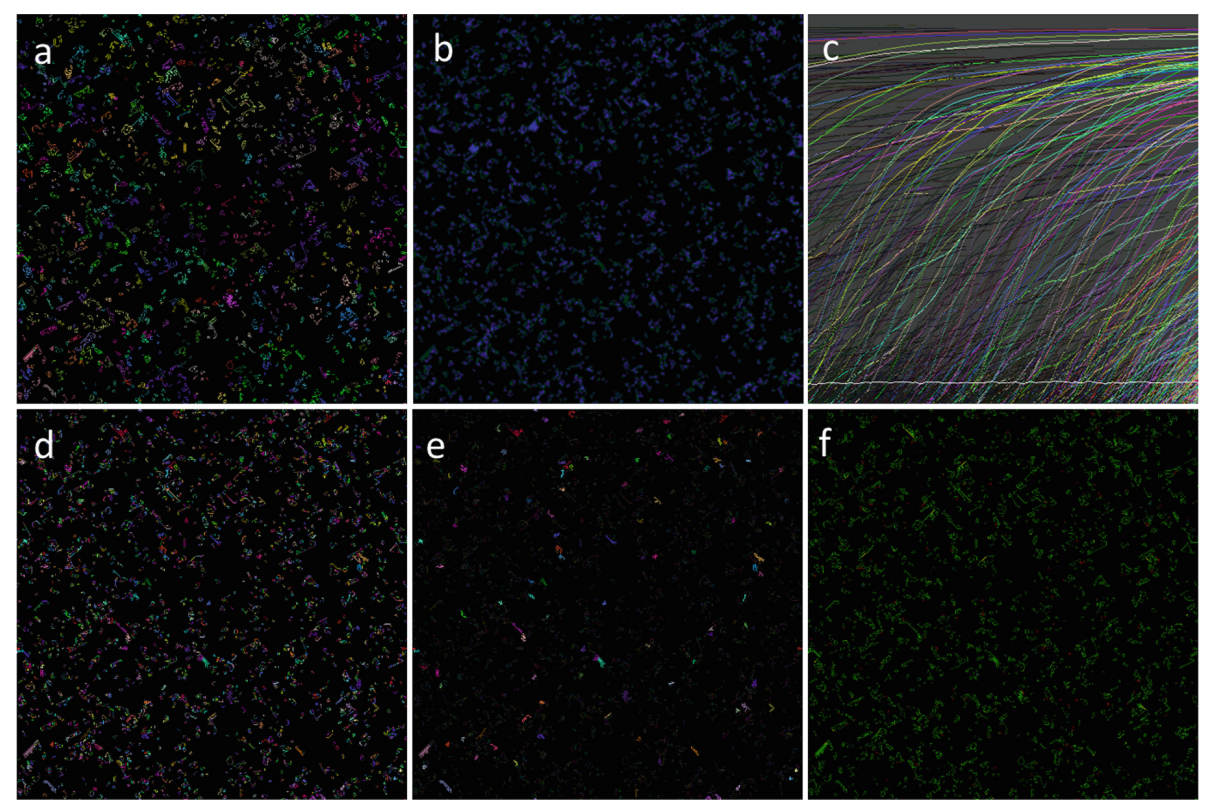

Fig. 10. Selection for longer period displacement histories resulting in rich populations of spaceships: complementary images at $t=12772$. (a) colouring individual genomes by ID (b) visualizing the neighbour states for survival (blue) and birth (green), with brightness proportional to s (c) activity statistics of last 512 time steps (d) connected components (genetically distinguished) serving as the basis for pattern analysis (e) only novel patterns (occurring for first time as this step) retain bright colours, all others darkened (f) colour coding displacements genealogies: quality of periodicity is brightness, with static period 1 entities in red and longer periods in increasing blue. (Color figure online)

\section{Discussion and Conclusions}

We have created genelife, a new version of Conway's Game of Life that is evolutionary, in the sense that all live cells are endowed with a genome that can be passed on, possibly changed by mutation, to offspring during birth processes. The GoL is an intriguing version of active media because it has proven capability for universal 
computation, but computation does not arise in typical asymptotic dynamics on finite lattices. Genelife's evolutionary version of the GoL seeks to capitalize on the implicit computational capabilities of the GoL to provide an evolutionary system that can produce computation as an emergent property of the evolutionary dynamics.

One feature of cellular automata with complex dynamics that has been historically important is that the medium should be active, but have a natural relaxation to a quiescent state, as indeed is observed for the GoL. Only special and rare initial conditions lead to ongoing complex dynamics in the GoL, including expansive dynamics that can escape any finite bounded region, and these initial conditions are considered to be the carriers of complex natural (including universal) computation. Bays [59], for example, in his search for GoL-like rules in $3 \mathrm{D}$, specifies that a rule R defines a "Game of Life" if and only if both of the following are true:

1. A glider must exist and must occur "naturally" if we apply R repeatedly to primordial soup configurations. (A primordial soup is any finite mass of arbitrarily dense randomly dispersed living cells.)

2. All primordial soup configurations, when subjected to $\mathrm{R}$, must exhibit bounded growth.

In genelife, we could readily attain this criterion (or some more precise version of it) by making genes rare in sequence space that encode local rule departures that give rise to a live cell by a proper birth process (i.e. not a movement event in which a parent live cell gives rise to only one offspring and dies at its previous location). As we have implemented it here, however, we have simply accelerated the process of discovery for genes that can modify the local birth processes. We investigated the first two steps in making such genes rarer for the semi-totalistic case via the parameter $n_{c}$ : increasing the number of bits to encode a LUT entry from 1 to 2 to 4 . We maintain that it is not natural to assume that the probability of configurations that may give rise to complex or universal computation is much lower than the probability of configurations that may lead to proliferation.

In some sense, our goal of having a rich evolving system contrasts with the desire of asymptotic quiescence characteristic of the GoL. We seek a medium that is active enough to spontaneously produce complex evolutionary dynamics rather than quiescence. But for evolutionary progress to be observed, we must still have a constraint on the activity of the medium, otherwise chaos ensues.

It is important to stress that many of the innovations presented here can be generalized beyond the framework of nearest neighbour and binary CAs. The use of nearest neighbours only in the CA rules is clearly just a convenient starting point for genetic extension models as proposed here. GoL models have been extended to larger neighbourhoods [60] and 3D [59], to provide more differentiated rules near to the GoL configurations $s=2,3$, and non-discrete generalizations such as Smooth Life [61] have also been proposed. Larger neighbourhoods in CAs do result in new phenomena, such as making spiral waves commonplace $[31,33]$. The insights and tools developed for genelife will be useful in the more general context of both less strictly local rules, other lattices and even continuous state dynamical systems such as coupled map lattices [62].

Likewise, our association of genomes with live states in binary CAs is compelling but is not a fundamental limitation of the domain of application of genetic extensions. 
Firstly, the above approach can be used directly whenever it makes sense to distinguish two classes of states: the inanimate or physical states (devoid of biological information that can be inherited) and the live states (containing copyable or inheritable information). Secondly, one can also add ancillary information, differentiating inanimate states, in the same way as for live cells. The simplest example of this is the association of information strings also with 0 states in the current model. This could be viewed as extending the GoL with a more complex environment with differentiated resources, or put more simply, as adding a chemistry to the GoL. While transitions which copy long strings of such information would be equivalent to biological replication, which is not a property as yet found spontaneously in synthetic chemistry, there are a wealth of autocatalytic chemical reactions for simple substances that are formally equivalent to copy processes for limited information content.

In conclusion, we may regard genelife as a success, in the sense that it provides a rich evolutionary platform with a variety of mechanisms to exert control over evolutionary processes. We have also developed a novel coherent set of analytical tools reflecting both genetic and spatial information processing. Success might be considered limited, in the sense that emergence of increasingly complex computation within a genelife population remains an empirical challenge. Genelife's ability to control evolution through constraints on genetic expression will enable the engineering of evolution's emergent properties. We look forward to exploring these directions in the future.

Acknowledgments. The authors would like to thank the Earth Life Science Institute (ELSI Tokyo, Japan) for hosting them for a short visit as part of an EON Seed Grant in 2017 where this work began. The EON Seed Grant was supported by the John Templeton Foundation. Our thanks also to Nathaniel Virgo and Steen Rasmussen for helpful comments at an early stage in this work.

\section{Supplementary Material}

\section{Deterministic Resolution of Neutral Selection}

In general, if two genomes are different and a neutral outcome is sought, then some other mechanism must be invoked to choose an ancestor for the newly born genome. The conventional population genetics approach of choosing one of them randomly adds a major source of stochasticity to the otherwise deterministic GoL. It turns out there are a number of possible alternatives:

1. Random choice of live neighbours for birth

2. Distinguish live neighbours for birth by their position in the configuration

3. Examine the neighbourhoods of live neighbours to distinguish them.

Both 2. and 3. suffer from potential ambiguity if the live neighbours remain identical under the distinction. We obviously would wish to preserve a certain degree of spatial symmetry in both the alternatives 2. and 3. In the Supplementary Material figures Supp. Figs. 1, 2, and 3, we catalogue and illustrate the different configurations of live neighbours for the non-trivial cases of $\mathrm{s}=2,3,4$. The cases $\mathrm{s}=0,1$ are very simple by comparison, and the cases $5,6,7,8$ can be obtained simply by exchanging 
zeros and ones in the figures. For the GoL B3 rule, we note that there is a very simple generic principle for choosing a single ancestor among the three live neighbours positionally, and one that does not break any of the spatial symmetries considered: choose the one at the "most different" position. This most different position is indicated in green in the figure in the appendix. Generally, it turns out that two of the three positions are related to each other by more symmetries than the different one. Now this is very good news, because it means that a deterministic inheritance scheme for neutral selection based on spatial position can be achieved without breaking spatial symmetries. Because approach 3. is incomplete for the many cases when the live neighbours themselves have equal numbers of live neighbours, and because a realization of 2 . that works for B3 has been found, we do not pursue 3. further in this paper.

A somewhat weaker, but still valid procedure that generalizes 2 . to other numbers of live neighbours (e.g. 2, 4, etc.) is to recognize that the choice of the most different position for B3 can be broken down into three steps: (a) find a canonical representation of the pattern of live neighbours which represents all symmetric versions of the pattern (under one of the chosen symmetries above) (b) specify the absolute position of the chosen position in this canonical representation (c) transform this position relative to the canonical representation back to the "orientation" of the particular starting configuration. It turns out that since the canonical rotation is mapped symmetrically to each possible instance that even making a simple choice such as the first position in the canonical representation gives rise to a positional inheritance rule with symmetry preserving properties. However non-trivial genetic dynamics such as genetic rotors for GoL oscillators or still lifes are possible.

We illustrate this principle first for the case of octavian symmetry (Sect. 2.4), which turns out to play a pivotal role in the analysis, and then extend it to other (more physical) symmetries. The distinguished configurations for $\mathrm{s}=2,3,4$ are shown in the left column of figures A1-A3 for the 4,7,10 canonical rotations. These are simply and efficiently defined as the 8-rotation of the 8-bit binary pattern of live neighbours that has the smallest numerical value. All the different configurations for $\mathrm{s}=0-8$ live neighbours given by the binomial coefficients ${ }^{8} \mathrm{C}_{\mathrm{s}}(1,8,28,56,70,56,28,8,1)$ reduce to $(1,1,4,7,10,7,4,1,1)$ configurations distinguishable up to 8-rotation symmetry. Note that these numbers only differ from ${ }^{8} \mathrm{C}_{\mathrm{s}} / 8$ for $\mathrm{s}=0,2,4,6,8$ and because of the 6 ambiguous canonical bit patterns 00000000, 00010001, 00110011 and 01010101, 01110111 , and 11111111 (i.e. patterns that can be rotated into themselves with less than 8 single steps). For these patterns only, an alternative rule must be found to choose the ancestor if we allow B0, B2, B4, B6 or B8 rule extensions. In our implementation for these special cases, we coded the following 8 disambiguation options which are mostly deterministic but include one spatially and one genetically random option:

1. random choice: this involves a departure from determinism for these cases only

2. ignore problem and choose selected bit of canonical configuration: accepting minimal asymmetry induced by these comparatively infrequent (for s $=1-7$ ) cases.

3. disallow birth: this effectively modifies the rules and is like excluding these rules from the table

4. choose lesser in value of genes if different (otherwise it makes no difference) i.e. revert to non-neutral genetic model in these (rare) cases only 
5. similar to 4, choose gene with least number of ones and if same, then lesser in value

6. choose a recombinant AND of all genes involved in this case

7. choose a default ancestor such as the gene coding for the Game of Life in these cases only

8. generate a random gene to give birth to for these ambiguous instances.

The option 6 is potentially minimally disruptive, effectively just reducing the rate of departure from GoL dynamics and is better in most circumstances than option 5 that is also symmetric but creates a non-trivial correlation between dynamics and genetic change. However, depending on the investigation, each of the techniques has its strengths and weaknesses.

\section{2-live-neighbour configurations}
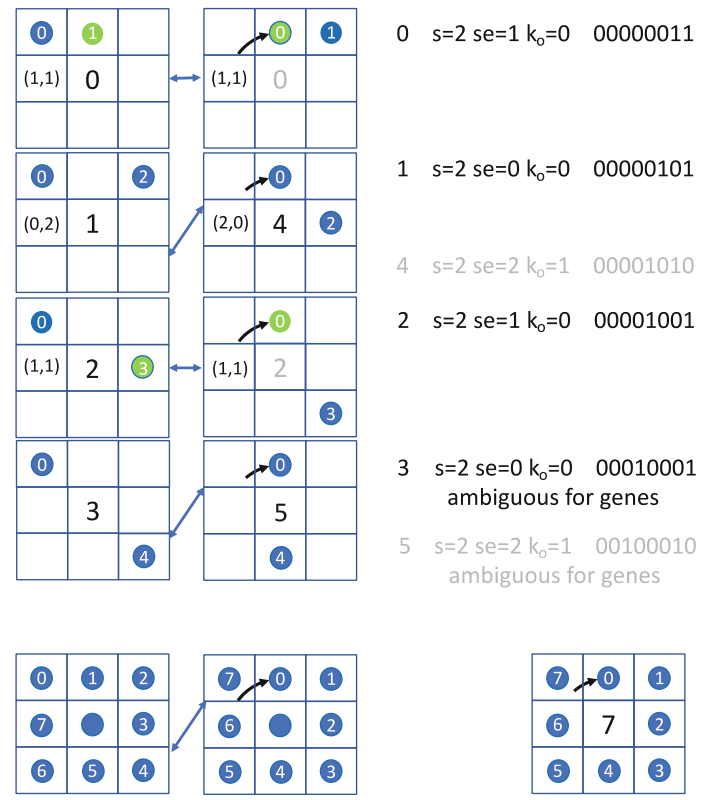

Supp. Fig. 1. Live neighbour configurations with $s=2$. The figure shows the configurations distinguished in the four different symmetries, in their relation to the canonical octavian symmetry, which distinguishes the four in the left column: semi-totalistic (1: i.e. all equivalent), quarter-totalistic (3: different se), octavian (4) and isotropic (6). The canonical minimal binary string representations of the configurations are shown on the right. The deterministic positional choice of ancestor is shown in green, when this is possible without further information (see main text). In the bottom row, the numbering scheme for neighbours is shown, along with its rotation. (Color figure online) 


\section{3-live-neighbour configurations}

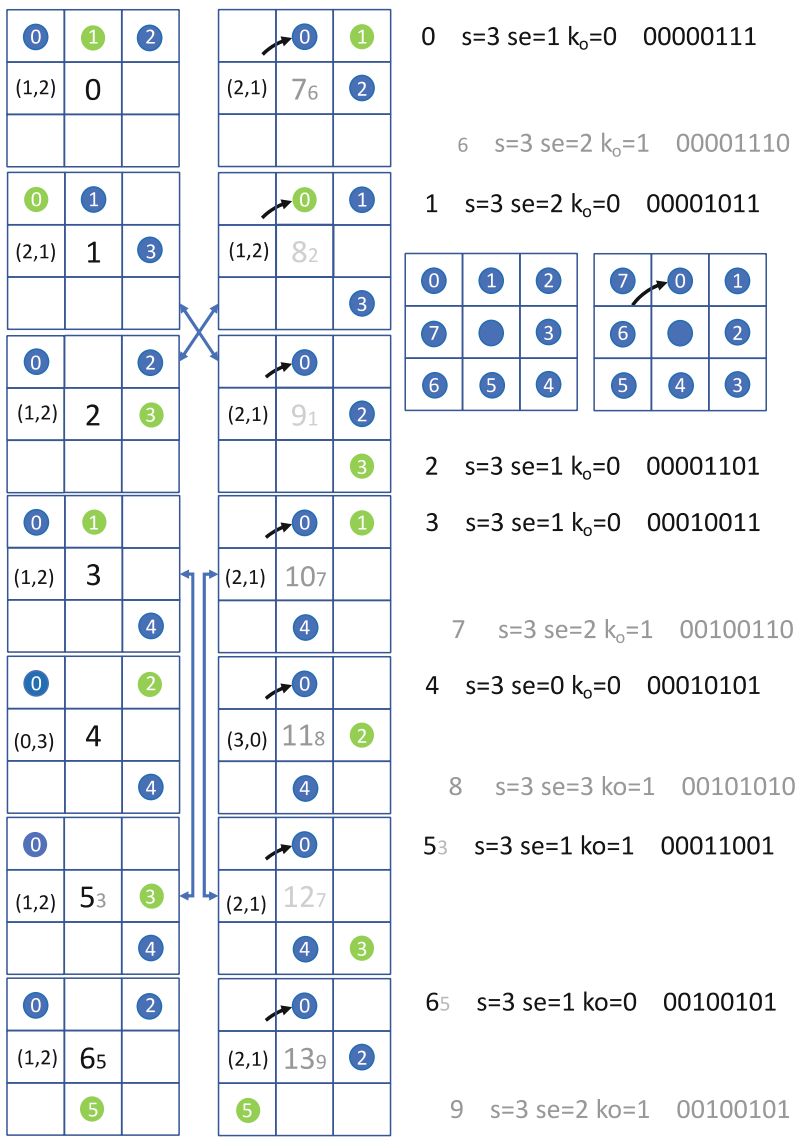

Supp. Fig. 2. Live neighbour configurations for $s=3$. Explanation is analogous to Supp. Fig. 1. The six distinguished configurations in octavian symmetry are shown in the first column. The distinction of corner and edge-centred neighbours raises this number to 10 in the fully isotropic case and only distinguishes these (by se) results in 4 different structures. Note that in this case, as for $s=5$ there is always a unique deterministic positional choice of neighbour, shown in green: the most different position (the other two have greater symmetry). (Color figure online) 


\section{4-live-neighbour configurations}

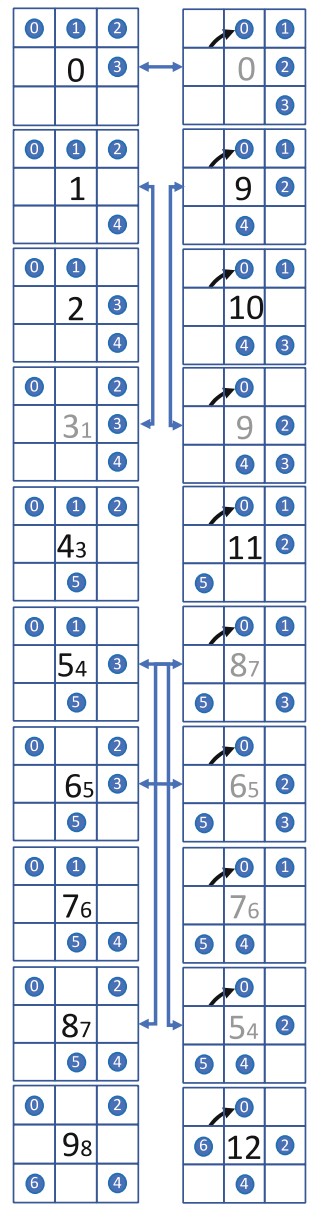

$0 \quad s=4$ se $=2 k_{0}=0 \quad 00001111$

$1 s=4 s e=1 k_{o}=0 \quad 00010111$

$9 \mathrm{~s}=4 \mathrm{se}=3 \mathrm{k}_{0}=1 \quad 00101110$

$2 \mathrm{~s}=4 \mathrm{se}=2 \mathrm{k}_{\mathrm{o}}=0 \quad 00011011$

$10 \mathrm{~s}=4 \mathrm{se}=2 \mathrm{k}_{\mathrm{o}}=1 \quad 00101110$

$3 s=4$ se $=1 k_{0}=0 \quad 00011101$

$4 \mathrm{~s}=4 \mathrm{se}=2 \mathrm{k}_{\mathrm{o}}=0 \quad 00100111$

$11 s=4 \mathrm{se}=2 \mathrm{k}_{\mathrm{o}}=101001110$

$5 \mathrm{~s}=4 \mathrm{se}=3 \mathrm{k}_{\mathrm{o}}=0 \quad 00101011$

$6 s=4 \mathrm{se}=2 \mathrm{k}_{0}=0 \quad 00101101$

$7 \mathrm{~s}=4 \mathrm{se}=2 \mathrm{k}_{\mathrm{o}}=0 \quad 00110011$

$8 s=4$ se $=1 k_{0}=0 \quad 00110101$

$9 \mathrm{~s}=4 \mathrm{se}=0 \mathrm{k}_{\mathrm{o}}=0 \quad 01010101$

$12 \mathrm{~s}=3 \mathrm{se}=4 \mathrm{k}_{\mathrm{o}}=1 \quad 10101010$

Supp. Fig. 3. Live neighbour configurations for $s=4$. Here there are 10 distinguished configurations in the octavian symmetry, 5 in quarter-totalistic and $13(0-12)$ in isotropic symmetry. The configurations 7 and 9(12) are the ones where it is not possible to unambiguously specify a particular choice of ancestor in way which is preserved under the symmetries and identifiable from the canonical binary representation. For all others a deterministic positional choice may be made. 


\section{Simulation Examples}

Supplementary figures Supp. Figs. 4 and 5 are movies that show the Game of Life dynamics, including genome activity (Supp. Fig. 4) and genealogy evolution (Supp. Fig. 5). Note that the population is evolving, in the sense that new genes are introduced into the population (through mutation), but the genes are totally uncoupled from the dynamics. They are simply "going along for the ride". Supp. Fig. 6 is referenced in Sect. 4.3.

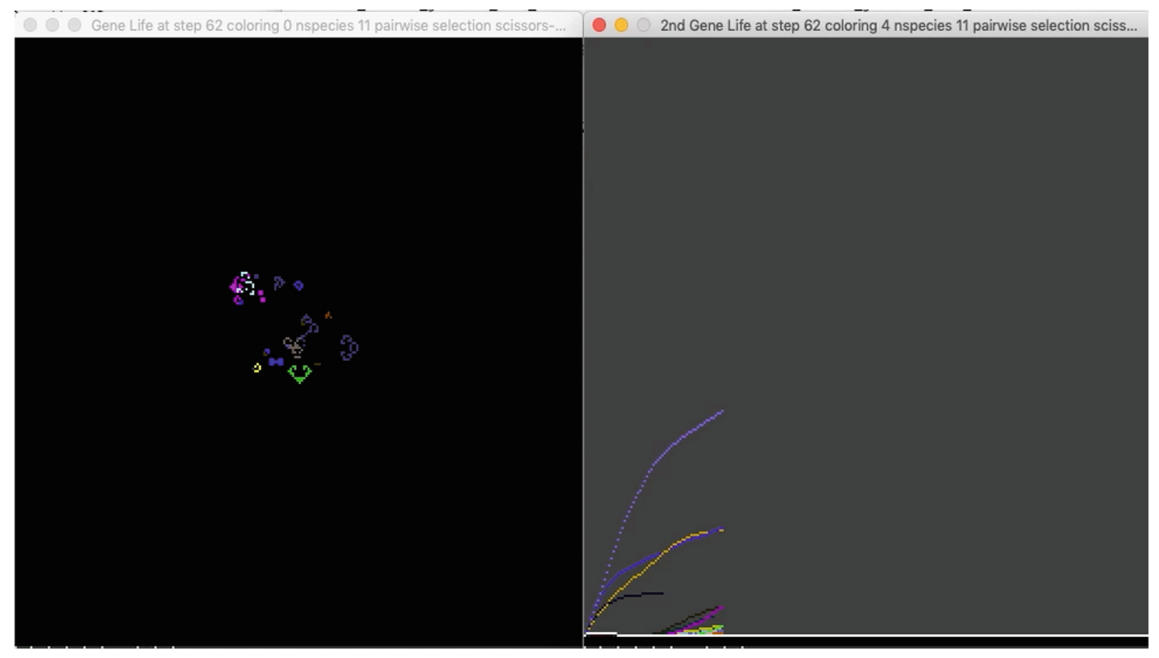

Supp. Fig. 4. Game of life time evolution, with activity.

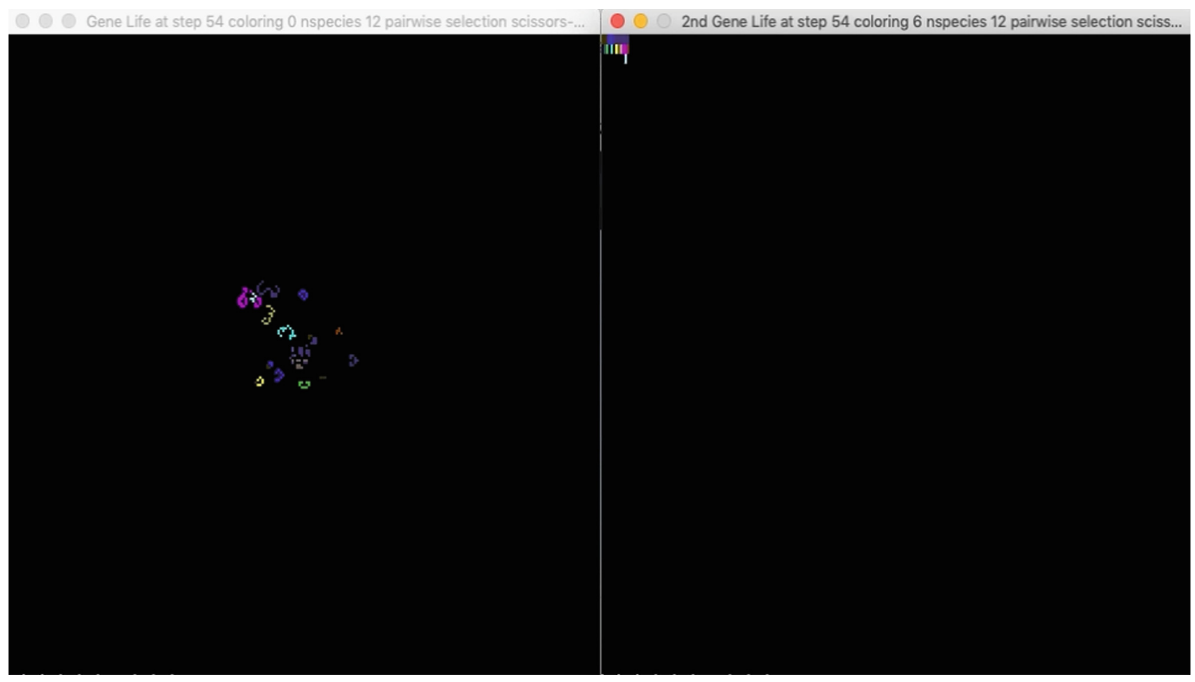

Supp. Fig. 5. Game of Life time evolution, with genealogies. First ancestor genealogies with the vertical axis genealogical depth, coloured by sequence type. 


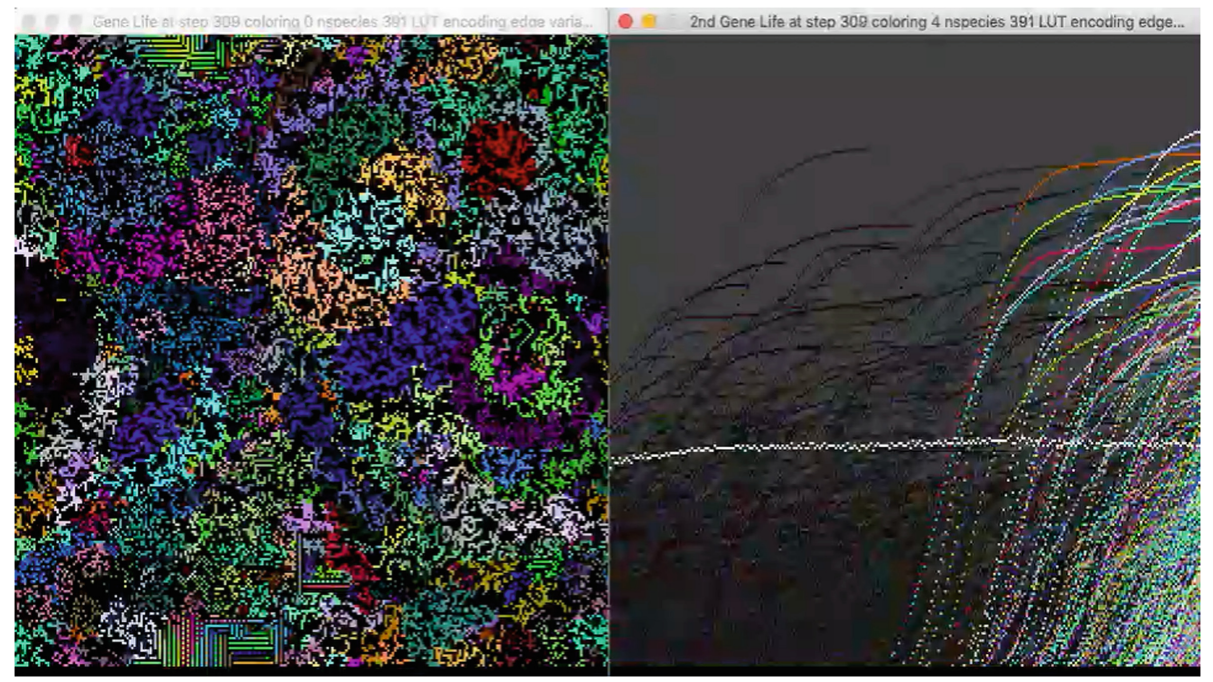

Supp. Fig. 6. Complex evolution discussed in Sect. 4.3 First phase: expanding blooms of competing domains, second phase is a collapse of the population, as it is almost taken over by a single genome, and the third phase is successful invasion of several new species, which continue to evolve for competing domains that have different dynamics from the original expanding bloom domains.

\section{References}

1. Von Neumann, J., Burks, A.W.: Theory of Self-reproducing Automata. University of Illinois Press, Urbana (1966)

2. Gardner, M.: Mathematical games -the fantastic combinations of John Conway's new solitaire game "life". Sci. Am. 223, 120-123 (1970). https://doi.org/10.1038/scientificamerican1070120

3. Wolfram, S.: Statistical mechanics of cellular automata. Rev. Mod. Phys. 55, 601-644 (1983). https://doi.org/10.1103/revmodphys.55.601

4. Packard, N.H., Wolfram, S.: Two-dimensional cellular automata. J. Stat. Phys. 38, 901-946 (1985). https://doi.org/10.1007/bf01010423

5. Rendell, P.: (2018). http://rendell-attic.org/gol/tm.htm

6. Pesavento, U.: An implementation of von Neumann's self-reproducing machine. Artif. Life 2, 337-354 (1995). https://doi.org/10.1162/artl.1995.2.4.337

7. Conway, J.H.: On Numbers and Games. A K Peters/CRC Press United States (2000). ISBN 9781568811277

8. Johnston, N.: (2019). http://www.conwaylife.com/wiki/LifeWiki

9. Berlekamp, E.R., Conway, J.H., Guy, R.K.: Winning Ways for Your Mathematical Plays. vol. 4, pp. 1004. A. K. Peters, Natick, Mass (2001). 1568811446

10. Rendell, P.: A fully universal turing machine in conway's game of life. J. Cell. Autom. 8, 19-38 (2013)

11. Bak, P., Chen, K., Creutz, M.: Self-organized criticality in the game of life. Nature 342, 780782 (1989). https://doi.org/10.1038/342780a0 
12. Bak, P.: Self-organized criticality in nonconservative models. Phys. A 191, 41-46 (1992). https://doi.org/10.1016/0378-4371(92)90503-I

13. Shapiro, A.: (2019). https://github.com/ShprAlex/SproutLife/wiki

14. Medernach, D., Kowaliw, T., Ryan, C., Doursat, R.: Long-term evolutionary dynamics in heterogeneous cellular automata. In: GECCO 2013: Proceedings of the 2013 Genetic and Evolutionary Computation Conference, pp. 231-238 (2013)

15. Ryan, C., Fitzgerald, J., Kowaliw, T., Doursat, R., Carrignon, S., Medernach, D.: Evolution of heterogeneous cellular automata in fluctuating environments. In: The 2019 Conference on Artificial Life, vol. 28, pp. 216-223 (2016). https://doi.org/10.1162/978-0-262-33936-0ch041

16. Zamaraev, A.: (2019). https://github.com/a5kin/evolife

17. Suzuki, K., Ikegami, T.: Homeodynamics in the game of life. In: The 2nd Annual Conference of the Japanese Society for Artificial Intelligence, pp. 600-607 (2008)

18. Taylor, T.: Redrawing the boundary between organism and environment. In: Artificial Life IX: Proceedings of the Ninth International Conference on the Simulation and Synthesis of Living Systems, pp. 600. The MIT Press (2004). https://doi.org/10.7551/mitpress/1429.003. 0045

19. Turney, P.D.: Modeling major transitions in evolution with the game of life. arXiv:1908. 07034 (2019)

20. Eppstein, D.: Growth and decay in life-like cellular automata. In: Adamatzky, A. (ed.) Game of Life Cellular Automata, pp. 71-97. Springer, London (2010). https://doi.org/10.1007/9781-84996-217-9_6

21. Mitchell, M., Hraber, P.T., Crutchfield, J.P.: Revisiting the edge of chaos: evolving cellular automata to perform computations. Complex Syst. 7, 89-130 (1993)

22. Schreiber, T.: Measuring information transfer. Phys. Rev. Lett. 85, 461 (2000)

23. Lizier, J.T.: Measuring the dynamics of information processing on a local scale in time and space. In: Wibral, M., Vicente, R., Lizier, J.T. (eds.) Directed Information Measures in Neuroscience. UCS, pp. 161-193. Springer, Heidelberg (2014). https://doi.org/10.1007/9783-642-54474-3_7

24. Helvik, T., Lindgren, K., Nordahl, M.G.: Local information in one-dimensional cellular automata. In: Sloot, P.M.A., Chopard, B., Hoekstra, A.G. (eds.) ACRI 2004. LNCS, vol. 3305, pp. 121-130. Springer, Heidelberg (2004). https://doi.org/10.1007/978-3-540-30479$1 \_13$

25. Crutchfield, J.P.: The calculi of emergence - computation, dynamics and induction. Phys. D 75, 11-54 (1994). https://doi.org/10.1016/0167-2789(94)90273-9

26. Crutchfield, J.P., Ellison, C.J., James, R.G., Mahoney, J.R.: Synchronization and control in intrinsic and designed computation: an information-theoretic analysis of competing models of stochastic computation. Chaos 20, 037105 (2010). https://doi.org/10.1063/1.3489888

27. Hanson, J.E., Crutchfield, J.P.: Computational mechanics of cellular automata: an example. Phys. D 103, 169-189 (1997). https://doi.org/10.1016/s0167-2789(96)00259-x

28. Marzen, S.E., Crutchfield, J.P.: Statistical signatures of structural organization: the case of long memory in renewal processes. Phys. Lett. A 380, 1517-1525 (2016). https://doi.org/10. 1016/j.physleta.2016.02.052

29. Shalizi, C.R., Crutchfield, J.P.: Computational mechanics: pattern and prediction, structure and simplicity. J. Stat. Phys. 104, 817-879 (2001). https://doi.org/10.1023/a:1010388907793

30. McCaskill, J.S., Packard, N.H., Rasmussen, S., Bedau, M.A.: Evolutionary self-organization in complex fluids. Philos. Trans. R. Soc. Lond. B Biol. Sci. 362, 1763-1779 (2007). https:// doi.org/10.1098/rstb.2007.2069 
31. Boerlijst, M.C., Hogeweg, P.: Spiral wave structure in pre-biotic evolution: hypercycles stable against parasites. Phys. D: Nonlinear Phenom. 48, 17-28 (1991). https://doi.org/10. 1016/0167-2789(91)90049-F

32. Nowak, M.A., May, R.M.: Evolutionary games and spatial chaos. Nature 359, 826-829 (1992). https://doi.org/10.1038/359826a0

33. Lindgren, K., Nordahl, M.G.: Evolutionary dynamics of spatial games. Phys. D - Spec. Issue Constr. Complex. Artif. Reality Arch. 75, 292-309 (1994). https://doi.org/10.1016/01672789(94)90289-5

34. Fraser, A.S.: An introduction to population genetic theory. By J. F. Crow and M. Kimura. Harper and Row, New York. 656 pp. 1970. Teratology 5, 386-387 (1972) https://doi.org/10. 1002/tera.1420050318

35. He, L., Ren, X., Gao, Q., Zhao, X., Yao, B., Chao, Y.: The connected-component labeling problem: a review of state-of-the-art algorithms. Pattern Recogn. 70, 25-43 (2017). https:// doi.org/10.1016/j.patcog.2017.04.018

36. Suzuki, K., Horiba, I., Sugie, N.: Linear-time connected-component labeling based on sequential local operations. Comput. Vis. Image Understand. 89, 1-23 (2003). https://doi. org/10.1016/S1077-3142(02)00030-9

37. Wu, K., Otoo, E., Suzuki, K.: Optimizing two-pass connected-component labeling algorithms. Pattern Anal. Appl. 12, 117-135 (2009). https://doi.org/10.1007/s10044-008-0109-y

38. Samet, H.: Connected component labeling using quadtrees. J. ACM 28, 487-501 (1981). https://doi.org/10.1145/322261.322267

39. Rokicki, T.: (2019). http://golly.sourceforge.net

40. Gosper, R.W.: Exploiting regularities in large cellular spaces. Phys. D: Nonlinear Phenom. 10, 75-80 (1984). https://doi.org/10.1016/0167-2789(84)90251-3

41. Volgenant, A.: Linear and semi-assignment problems: a core oriented approach. Comput. Oper. Res. 23, 917-932 (1996). https://doi.org/10.1016/0305-0548(96)00010-X

42. Gupta, R.: (2018). https://www.geeksforgeeks.org/hopcroft-karp-algorithm-for-maximummatching-set-2-implementation/

43. Liang, X.S.: Information flow and causality as rigorous notions ab initio. Phys. Rev. E 94 (2016). https://doi.org/10.1103/physreve.94.052201

44. Bossomaier, T.R.J.: An Introduction to Transfer Entropy: Information Flow in Complex Systems. Springer, Cham (2016). https://doi.org/10.1007/978-3-319-43222-9

45. Rupe, A., Crutchfield, J.P.: Local causal states and discrete coherent structures. Chaos 28 , 075312 (2018). https://doi.org/10.1063/1.5021130

46. Varn, D.P., Canright, G.S., Crutchfield, J.P.: Epsilon-machine spectral reconstruction theory: a direct method for inferring planar disorder and structure from X-ray diffraction studies. Acta Crystallogr. Sect. A 69, 197-206 (2013). https://doi.org/10.1107/s0108767312046582

47. Sinapayen, L., Ikegami, T.: Online fitting of computational cost to environmental complexity: predictive coding with the $\varepsilon$-network. In: The 2019 Conference on Artificial Life, vol. 29, pp. 380-387 (2017). https://doi.org/10.1162/isal_a_065

48. Gustavsson, M.: (2017). https://github.com/mattiasgustavsson/libs/blob/master/hashtable.h

49. Bedau, M., Packard, N.: Measurement of evolutionary activity, teleology, and life. In: Artificial Life II, pp. 431-461. Addison Wesley (1991)

50. Bedau, M.A., Snyder, E., Packard, N.H.: A classification of long-term evolutionary dynamics. In: From Anim Animat, pp. 228-237. MIT Press (1998)

51. Channon, A.: Unbounded evolutionary dynamics in a system of agents that actively process and transform their environment. Genet. Program Evolvable Mach. 7, 253-281 (2006)

52. Stout, A., Spector, L.: Validation of evolutionary activity metrics for long-term evolutionary dynamics. In: Proceedings of the 7th Annual Conference on Genetic and Evolutionary Computation, pp. 137-142. ACM (2005) 
53. Kingman, J.F.C.: The coalescent. Stochast. Process. Appl. 13, 235-248 (1982). https://doi. org/10.1016/0304-4149(82)90011-4

54. Blancas, A., Duchamps, J.J., Lambert, A., Siri-Jégousse, A.: Trees within trees: simple nested coalescents. Electron. J. Probab. 23. https://doi.org/10.1214/18-ejp219

55. Hein, J., Schierup, M.H., Wiuf, C.: Gene Genealogies, Variation and Evolution: A Primer in Coalescent Theory. Oxford University Press, Oxford and New York (2005). 0-19-852995-3

56. Brunet, É., Derrida, B.: Genealogies in simple models of evolution. J. Stat. Mech. (2013). P01006. https://doi.org/10.1088/1742-5468/2013/01/p01006

57. Kay, N.-S.: Graphs in sequence spaces: a review of statistical geometry. Biophys. Chem. 66, 111-131. https://doi.org/10.1016/s0301-4622(97)00064-1. PMID - 9362556

58. Smith, J.M., Szathmary, E.: The Major Transitions in Evolution. Oxford University Press (1997). ISBN 019850294X

59. Bays, C.: Candidates for the game of life in three dimensions. Complex Syst. 1, 373-400 (1987). ISSN 0891-2513

60. Evans, K.M.: Larger than life: it's so nonlinear. Ph.D. thesis University of Wisconsin, Madison (1996). http://www.csun.edu/ kme52026

61. Rafler, S.: Generalization of Conway's "Game of Life" to a continuous domain SmoothLife. arXiv:1111.1567 (2011)

62. Kaneko, K.: Overview of coupled map lattices. Chaos: Interdisc. J. Nonlinear Sci. 2, 279 282 (1992). https://doi.org/10.1063/1.165869

Open Access This chapter is licensed under the terms of the Creative Commons Attribution 4.0 International License (http://creativecommons.org/licenses/by/4.0/), which permits use, sharing, adaptation, distribution and reproduction in any medium or format, as long as you give appropriate credit to the original author(s) and the source, provide a link to the Creative Commons license and indicate if changes were made.

The images or other third party material in this chapter are included in the chapter's Creative Commons license, unless indicated otherwise in a credit line to the material. If material is not included in the chapter's Creative Commons license and your intended use is not permitted by statutory regulation or exceeds the permitted use, you will need to obtain permission directly from the copyright holder.

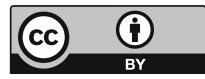

